\title{
Categorial Grammar and the Semantics of Contextual Prepositional Phrases
}

\author{
Nissim Francez ${ }^{*}$ and Mark Steedman ${ }^{\dagger}$
}

September 12, 2004

\begin{abstract}
The paper proposes a semantics for contextual (i.e., Temporal and Locative) Prepositional Phrases (CPPs) like during every meeting, in the garden, when Harry met Sally and where I'm calling from. The semantics is embodied in a multi-modal extension of Combinatory Categoral Grammar (CCG). The grammar allows the strictly monotonic compositional derivation of multiple correct interpretations for "stacked" or multiple CPPs, including interpretations whose scope relations are not what would be expected on standard assumptions about surface-syntactic command and monotonic derivation. A type-hierarchy of functional modalities plays a crucial role in the specification of the fragment.
\end{abstract}

\section{Introduction}

In [25], a temporal semantics for temporal preposition phrases (TPPs) was introduced, free of any syntactic analysis and syntax-semantics interface. This semantics employed some unorthodox operations, referred to as pseudoapplications, as well as semantic operations not triggered by any underlying syntactic manipulation. Furthermore, it used two types of sentential (and, hence, verbal) meanings. Here, we attempt to incorporate a semantics inspired by that of [25], but cast into the rigid syntax-semantics interface imposed by Combinatory Categorial Grammar (CCG, [27, 29]). In addition, we extend the semantics to two varieties of contextual preposition phrases (CPPs): these are the temporal preposition phrases (TPPs) of the earlier account, and Locative preposition phrases (LPPs), discussed in a related approach by [5], but not treated before in this framework. Of particular interest is mixed modification by both kinds of CPP. The fact that CCG is a strongly lexicalized grammatical theory allows us to bring all operations relating distinct sentential and verbal meanings under the control of lexical rules and morphemes such as tense inflections and the temporal prepositions themselves. We return to this issue in section 3.1.1. Finally, the theory is smoothly extended to (constituent and non-constituent) coordination of CPPs, as expected in a categorial approach, highlighting certain aspects of interpretation in such coordinations.

In spite of its rigidly type-driven character, CCG offers a larger repertoire of operations for manipulating meanings than just functional application. In particular, function composition in its various forms turns out very useful in this context, obviating the need for pseudo-applications. The involvement of type-raising also leads to certain simplifications. A type-hierarchy of functional modalities proposed by [2], following earlier versions proposed by [14], [20], and [19], plays a crucial role in the specification of the language fragment considered.

A central issue for this paper is the incorporation into the predicate-argument structure of contextual arguments, variables ranging over times (taken here as real intervals) of semantic type $i$, and locations (taken here as threedimensional regions) of semantic type $l .^{1}$

\footnotetext{
${ }^{*}$ Computer Science dept., the Technion-IIT, Haifa, Israel

${ }^{\dagger}$ School of Informatics, Edinburgh University, 2 Buccleuch pl., Edinburgh EH89LW

${ }^{1}$ The types $i$ and $l$ can either be be thought of as sub-types of the traditional type $e$ (of entities), with the proviso that they have fixed interpretations (that is, as real intervals or three-dimensional regions) in all models. Alternatively, they can be thought of as types distinct from $e$, again with the above interpretation in all models, in a move reminiscent of Gamut's [10], p.133-4 approach to intensionality.
} 
The distinguishing feature of such variables is that they do not correspond to any syntactic argument in the surface structure. As such, they never get values by being bound to meanings of other surface elements; rather, they are quantified over, abstracted over, and/or play a role in compositions, at the level of interpretation only. We return to this point below, and highlight some consequences. To emphasize the point, we use $x, y$ for arguments corresponding to syntactic complements, and $I, J, M, L$ for semantic arguments (here, contextual) not corresponding to syntactic complements. Furthermore, a predication of a predicate $p$ involving both kinds of arguments will be depicted, for example, as $p(x, y)(J, L)$, separating the arguments for readability. Note that we take here such predications as primitive. For a recent discussion of the nature of such predications in the temporal case (e.g., its relation to $p(x)\left(J^{\prime}\right)$, for $J^{\prime}$ a subinterval of $J$, see [7]).

The lexical meanings are responsible for the incorporation of contextual arguments (not originating from the syntax), and for some of the quantification over them, in addition to their other arguments that do originate from the syntax. We return to this issue in more detail in subsection 2.1, and in subsection 3.1.3 when discussing verb-meanings.

The semantics, in its CCG-interface, has to cope with the following issues.

- Both TPP-modification and LPP-modification should be naturally cascadable, as follows.

(1) Mary kissed John.

(2) Mary kissed John in every meeting.

(3) Mary kissed John in every meeting during some summer.

(4) Mary kissed john in some garden.

(5) Mary kissed John in some garden behind every tree.

A cascade of CPPs can also be preposed, as follows.

(6) In every meeting, Mary kissed John.

(7) In every meeting, during some summer, Mary kissed John.

(8) Behind every tree, Mary kissed John.

(9) In some garden, behind every tree, Mary kissed John.

When interpreting such a cascade, whether temporal or locative, the order of CPP-modifications (determining the relative scope of the quantifiers in the CPP-meanings) need not necessarily coincide with the surface order of those CPPs.

- TPPs and LPPs can mix in their modification.

(10) Mary kissed John in some garden during every meeting.

Longer mixed cascades are possible too, also preposed.

- A CPP can associate to and modify either a sentence or a noun in a preceding CPP, as follows.

(11) Mary kissed John [tpp in every meeting] [tpp during some summer]

(12) Mary kissed John [t $t p$ in every [ $n$ meeting [tpp during some summer]]] Again, a mixture of extents modification is possible.

(13) Mary kissed John [tpp in every meeting] [lpp in some garden]

(14) Mary kissed John [tpp in every [ $n$ meeting [lpp in some garden]]]

(15) Mary kissed John before [a meeting in some summer in every conference].

- Some data about (constituent and nonconstituent) coordination within CPPs is presented in Section 6.

Informally, the semantic effect of a CPP-modification is captured by restricting the event-extents of the modified event (the meaning of the modified sentence) to be sub-interval/sub-region of the extents of the modifying event (the meaning of the CPP), or of functions thereof. 
Note that PP-modification like that in the examples below is excluded from our framework; though occasionally viewed as LPPs in the literature, they do not really modify an event location. Rather, they affect the location of a participant of the event, and as such should be treated as dative complements rather than LPP modifiers.

(16) Mary put the laptop on a desk.

(17) A satellite was launched into the atmosphere.

We refer the reader to [25] for a detailed discussion of the relationship of the proposed (temporal) semantics to previous work. However, as we were finishing the current paper, we came across [26], which adopts a very similar (temporal only) semantics, presented in a GB syntactic framework. It also combines TPP modification with tense and aspect in an intuitively appealing way, neither of which are fully treated here or in [25]. We return to [26] in section 3.1.1.

\section{CCG}

Combinatory Categorial Grammar (CCG) is a strongly lexicalized grammar formalism. The CCG lexicon assigns each terminal symbol (that is, each word in the case of natural language) a finite set of categories. These categories are drawn from a set that can initially be defined in terms of some finite set $\mathcal{B}$ of primitive categories (the primitive categories used here are $\mathcal{B}=\{N P, N, S\}$ ), as follows:

(18) a. If $\alpha$ is a primitive category, then $\alpha$ is a category.

b. If $\alpha$ and $\beta$ are categories, then $\alpha / \beta$ and $\alpha \backslash \beta$ are categories.

As usual, nothing else is a category.

For example, in the notation used here, the category of a transitive verb such as kiss might be written as follows: ${ }^{2}$

(19) $\llbracket$ kiss $\rrbracket=(S \backslash N P) / N P: \lambda x^{e} \lambda y^{e} . \mathbf{k i s s}(x, y)$

The category itself can be thought of as defining a directionally specified function, with the category to the right of a slash defining the syntactic type of an argument, and that to the left defining the syntactic type of the result. ${ }^{3}$

The colon ":" pairs the category with an interpretation. Such interpretations (i.e., predicate-argument structures) are represented as usual by $\lambda$-terms, expressions in some higher-order language akin to (the extensional sublanguage of) Montague's $I L$. When presenting $\lambda$-terms, we include the type of the abstracted variable as a superscript in the abstraction itself, but omit it in the body of the term.

In what follows, we will overload the term "category" to refer both to the original purely syntactic definition, and to the pairing of such a category with an interpretation.

The lexicon used here will become apparent as the theory unfolds, and is summarized in an appendix.

Categories combine with other categories by Combinatory Rules. Figure 1 displays the Combinatory Rules of Functional Application and Composition that are used in the present paper. ${ }^{4}$ Here $\mathbf{B}$ labels the composition rules, since it is the identifier of the composition combinator in [6], where $\mathbf{B} f g \equiv f \circ g \equiv \lambda x . f(g(x))$ for $f, g$ of any composable function types. Note that the combinatory rules are polymorphic. The metavariables $X, Y, Z \ldots$ range over syntactic categories.

The inclusion of the rules $>\mathbf{B}_{\times}$and $<\mathbf{B}_{\times}$of "crossed" composition, in which the directionality of the operands are different, distinguishes CCG from the (weakly context-free) Lambek calculus, and is one of the factors that makes it "mildly context sensitive" ([16]). We will see below that such crossed composition rules are required to capture the syntax and semantics of adjuncts in English. However, such rules have an "order-permuting" effect, and in a

\footnotetext{
${ }^{2}$ Boldface, as in kiss indicates a semantic constant, which in other version of the theory have been indicated with primes, as in kiss'

${ }^{3}$ This notation is therefore the "result leftmost" variant. There is an alternative "result on top" notation, due to [17]. The latter is harder to read when "crossed" combinatory rules are involved, as here.

${ }^{4}$ A third species of combinatory rule related to the "substitution" combinator $\mathbf{S}$ is omitted from the present discussion.
} 


$$
\begin{gathered}
\frac{X /_{\star} Y: f \quad Y: g}{X: f(g)}>\frac{Y: g \quad X \backslash_{\star} Y: f}{X: f(g)}< \\
\frac{X /_{\diamond} Y: f \quad Y /_{\diamond} Z: g}{X /_{\diamond} Z: \lambda z \cdot f(g(z))}>\mathbf{B} \frac{Y \backslash_{\diamond} Z: g \quad X \backslash_{\diamond} Y: f}{X \backslash_{\diamond} Z: \lambda z \cdot f(g(z))}<\mathbf{B} \\
\frac{X /_{\times} Y: f \quad Y \backslash_{\times} Z: g}{X \backslash_{\times} Z: \lambda z \cdot f(g(z))}>\mathbf{B}_{\times} \frac{Y /_{\times} Z: g \quad X \backslash_{\times} Y: f}{X /_{\times} Z: \lambda z \cdot f(g(z))}<\mathbf{B}_{\times}
\end{gathered}
$$

Figure 1: The Modal CCG rules

language like English must be very severely constrained, either by limiting the combinatory rules themselves, as in [29], or by "modalizing" the categories in relation to the rules, as we do here, following [2].

Figure 1 follows [2,3] in assuming that function categories are "modalized," as indicated by a subscript on their slash, and that slash modalities are features in a type hierarchy, drawn from some finite set $\mathcal{M}$ (the modalities used here are $\mathcal{M}=\{\star, \diamond, \times, \cdot\})$. The most general type "." of slash subsumes two more specialised types " $\diamond$ " “ $\times$ ", which in turn subsume the most restricted type " $\star$ ”, as in Figure 2. The crossed composition rules are restricted

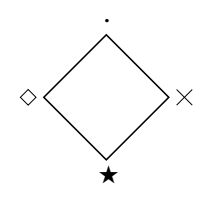

Figure 2: CCG Functional Type-hierarchy (adapted from Baldridge 2002).

to categories which are compatible with a "permuting" modality indicated by the $\times$ subscript annotation on the slashes in the rules. 5

We therefore rewrite the definition (18) of categories as follows:

a. If $\alpha$ is a primitive category, then $\alpha$ is a category.

b. If $\alpha$ and $\beta$ are categories, then $\alpha / \mu$ and $\alpha \backslash_{\mu} \beta$ are categories, where $\mu \in \mathcal{M}$.

Slashes in lexical categories also bear modalities, which limit the rules which can apply to them. The most permissive slashes /. and \. will be abbreviated as plain slashes, / and \respectively. We can therefore continue to write the category of the transitive verb as in (19). Since they subsume the slashes of type $\times$, categories bearing this most general type can combine by the crossed rules. However, neither categories bearing the slash types /。 and $\downarrow_{\diamond}$, nor those bearing the most restrictive types $/_{\star}$ and $\backslash_{\star}$, can combine by crossed rules. For example, the relative pronoun category used in example (23) below bears the modality $\diamond$ and can compose via the non crossed rules but not the crossed. The conjunction category discussed in section 6 bears the even more restrictive $\star$ modality, and can only apply. We will see later how these restrictions limit overgeneralization.

CCG allows also generalized composition. For example it includes the following rule, where $\mathbf{B}^{2} f g \equiv \lambda w \lambda z . f(g(w, z))$ :

$$
\frac{X / \Delta Y: f \quad(Y / \Delta Z) / \Delta W: g}{(X / \Delta Z) / \Delta W: \lambda x \lambda y \cdot f(g(x, y))}>\mathbf{B}^{2}
$$

\footnotetext{
${ }^{5}$ [2] presents this hierarchy the other way up, viewing it as a hierarchy of increasingly general rules and the ever more inclusive classes of categories to which they apply.
} 
The rules $>\mathbf{B}^{3},<\mathbf{B}^{2},<\mathbf{B}_{\times}^{2}$, etc. are defined analogously. While it is sometimes convenient to abbreviate generalized forward composition, say, as $>\mathbf{B}^{n}, n$ is bounded and in fact there is no compelling linguistic reason to allow $n$ greater than around 4.

Another generalized rule, for coordination, is deferred to Section 6.

Combinatory Categorial Grammars also allow operations of type-raising, corresponding to the combinator $\mathbf{T}$, where $\mathbf{T} a \equiv \lambda f . f(a)$. This operation turns a specified set of argument categories (notably, NP) into second-order functors over those functors (such as verbs) of which they are arguments. For example, an NP like Mary can turn into a functor over predicates, with the following category: ${ }^{6}$

(21) $\llbracket \mathrm{Mary} \rrbracket=S /(S \backslash N P): \lambda p^{(e t)} \cdot p($ mary $)$

The effect is as if Mary bore a nominative case inflection of the kind found in Latin. Of course, case in this sense is usually ambiguous in English, unlike Latin: Mary can also take the following "accusative" category, mapping transitive verbs into intransitive VPs:

$$
\llbracket \mathrm{Mary} \rrbracket=(S \backslash N P) \backslash((S \backslash N P) / N P): \lambda p^{(e(e t))} \lambda y^{e} . p(\mathbf{m a r y}, y)
$$

In both cases, the $\lambda$-term to the right of the colon identifies the logical form of the verb.

CCG derivations are notated as in the following example of a relative clause:

$$
\begin{aligned}
& \frac{\operatorname{man}}{N: \lambda x^{e} \cdot \operatorname{man}(x)} \frac{\text { that }}{\left(N \backslash_{\diamond} N\right) /(S / N P): \lambda p^{(e t)} \lambda q^{(e t)} \lambda y^{e} \cdot q(y) \wedge p(y)} \frac{\text { Mary }}{\frac{S /(S \backslash N P): \lambda r^{(e t)} \cdot r(\text { mary })}{(S \backslash N P) / N P: \lambda z^{e} \lambda w^{e} \cdot \mathbf{k i s s}(z, w)}} \\
& S / N P: \lambda z^{e} \cdot \mathbf{k i s s}(z, \text { mary }) \\
& N \backslash_{0} N: \lambda q^{(e t)} \lambda y^{e} \cdot q(y) \wedge \mathbf{k i s s}(y, \text { mary }) \\
& N: \lambda y^{e} \cdot \operatorname{man}(y) \wedge \text { kiss }(y, \text { mary })
\end{aligned}
$$

This treatment of extraction immediately generalizes to unbounded relativization and right node raising, capturing many other extraction and coordination phenomena including various forms of "nonconstituent" coordinations and restrictions on extraction attributed to the Empty Category Principle ([27, 29]).

Since type-raising, viewed as case, can in English as in Latin be regarded as a process of the morpho-lexicon rather than syntax (albeit a much more ambiguous one than in the latter language), and since we do not wish to burden the reader with syntactic notation, or distract them by this categorial ambiguity, we will not distinguish between (nominative, accusative, etc.) type-raised versions of categories like $N P, N P / N$, and the like, writing them indiscriminately as $N P \uparrow, N P \uparrow / N$, and so on (the up-arrow as a reminder that these are in fact second-order functional categories).

Since the present paper is primarily semantic in focus, the reader is directed to [27, 29, 2] and authors cited therein for detailed motivation in terms of syntax and semantics of coordination, relativization, and intonation structure in English and other languages for CCG and its specific inventory of combinatory rules.

\subsection{The contextual variables problem}

In order to deal with temporal/spatial adjuncts like in every meeting, in the garden, when Harry met Sally or where I'm calling from, we must consider sentences as having (temporal and locative) extents, and temporal and spatial adjuncts as functions over such extents.

We will show that to do this with the generality demanded by the observations in Section 1, it is necessary to identify propositions with functions over event-extents, of type $(i(l t))$, where $i$ (for interval) is the type of a temporal extent and $l$ (for location) is a type of a spatial extent, rather than individuals of type $t$ (i.e., truth-values) as is

\footnotetext{
${ }^{6}$ Slashes in type-raised categories take their type from that in the function they combine with-that is, their type is a variable. Strictly speaking this is distinct from the most general slash-type ".". However, we will abbreviate both as a plain slash here.
} 
traditional. We will further show that it is necessary to identify the meaning of a tensed clause of type $S$ with that of an "contextual modifier" of type $7((i(l t))(i(l t)))$.

This means that the one-to-one correspondence between semantic arguments and syntactic ones exhibited in traditional categories like (19) cannot be maintained.

However, we can keep these extra arguments out of the way of the syntactic derivation by ordering them after the syntactically realized ones, and making functions over sentences into functions over this functional type. Thus intransitive verbs will be of type $(e((i(l t))(i(l t))))$ (rather than $(e t))$, while VP adjuncts will be $((e((i(l t))(i(l t))))(e((i(l t))(i(l t)))))$ (rather than $((e t)(e t)))$.

Since $((i(l t))(i(l t)))$ plays much the same role in these categories as $t$ does in Montague's theory, we can ease the notational strain on the reader by abbreviating the former category as $\vec{t}$, writing intransitive verbs as $(e \vec{t})$ and VP adjuncts as $((e \vec{t})(e \vec{t}))$. Noun-phrases get a similarly extended denotation as Generalized Quantifiers of type $((e \vec{t}) \vec{t})$

In short, we will obtain our types (with the exception of the noun type $(e(i(l t))))$ by systematically replacing the type $t$ in standard types by $\vec{t}$, short for $((i(l t))(i(l t)))$.

To paraphrase Lewis' observation concerning simplicity ([18])—where we promised truth, we deliver functions from functions from event-extents to truth to functions from event-extents to truth. In the next section we show that this type-inflation is both necessary and desirable, because it allows us to assign some novel types to modifiers to support a correct semantics of nominal and sentential contextual modification.

\section{Cascading modification of CPPs}

In this section, we develop the part of the formalism that allows cascading CPP-modification. For simplicity of presentation, we first consider the case where the cascade of CPPs is entirely sentence-final.

\subsection{Ordered cascading}

We start by considering the interpretation of cascaded CPPs in their surface order of appearance.

For ease of presentation, we find it convenient to assume that CPP-modification is sentential modification, occurring after the verb has combined with its complements (including the subject). The changes needed for viewing CPPs as VP-modifiers are presented at the end of 3.1.5. The sentences considered here can be seen (at least as a first approximation) as "event-reporting" sentences. (We intend to treat "state-reporting" sentences elsewhere.) Events here are taken to have a pair of event-extents: an event-time $J$ and an event-location L.

Sentences are of category $S$, and their meanings are contextual Modifiers having the form $\lambda P^{(i(l t))} \lambda I^{i} \lambda M^{l} . \phi(P, I, M)$ and semantic type $((i(l t))(i(l t)))$ (or $\vec{t}$ for short), in which the event-time and event-location are both existentially quantified over (but see the discussion below regarding different quantifications), and are included within the respective contextual arguments. For example,

$$
\text { 『Mary kissed John } \rrbracket=S: \lambda P^{(i(l t))} \lambda I^{i} \lambda M^{l} \exists J^{i} \exists L^{l} \cdot \mathbf{k i s s}(\mathbf{j o h n}, \mathbf{m a r y})(J, L) \wedge J \subseteq I \wedge L \subseteq M \wedge P(J, L)
$$

This meaning can be paraphrased as within the contextual extents, there exists an event-time and an event-location, during which a kissing event (of John by Mary) took place. ${ }^{8}$

Recall that sentences have a dual function: they can both be modified by a CPP, and also serve as a preposition complement in a CPP. The role of the $I$ and $M$ variables is to serve as contextual extents, within which the eventextents will always be located. ${ }^{9}$ The semantic function of CPP-modification will be to restrict $I$ and $M$ to fall

\footnotetext{
${ }^{7}$ A referee has asked how is this type related to the "dynamic" treatment in [?]. According to Muskens (private communication), the correct relationship is obtained by observing that his type $s$ can be viewed as encoding the list of types $(i, l)$. Then, one observes that, in programming languages terms, our type $((s t)(s t))$ is a predicate transformer type (used also in update semantics [? ]), while Muskens' type $(s(s t))$ is a type of a state transformer (used also in DPL [? ]). There are well-known mutual translations between predicate and state transformers.

${ }^{8}$ We use the relation $\subseteq$ to denote the "part of" relation between spatio-temporal intervals. We do not assume or imply that such intervals are actually sets.

${ }^{9}$ These event-extents are related to a generalization of Reichenbach's "reference time" R, in the following senses. First, Reichenbach
} 
within the range of quantification of the CPP-quantifiers on their own $J$ and $L$ (or functions thereof), as we'll see below. Thus, the contextual extents "implement" the function of a sentence as a CPP-modificand.

We note that in existentially quantifying over $J$ and $L$, we are simplifying in order to ease the notational burden on the reader. In fact, these event-extents are more like definites, or the generalized skolem-terms used in place of existentials in [30]. The present use of existentials has some minor undesirable consequences that we will point out as they arise, and should be taken as merely a placeholder for a more precise account.

The role of $P(J, L)$ in the sentential meaning will become clear when dealing with a sentence serving as a sentential complements of temporal prepositions, in Section 5. It will restrict the range of the the contextual extents of the matrix sentence (the modified one) to fall within the scope of the existential quantifiers of the event-extents in the $C P$-complement sentence. ${ }^{10}$

The parenthesization of the arguments emphasizes the point made earlier: the $x, y$ arguments (the subject and object, respectively, in the transitive case), correspond to syntactic arguments, when a verb is applied as a syntactic function to concatenated complements. On the other hand, the contextual $I, J, M, L$ arguments do not correspond to syntactic arguments.

We describe how sentence meanings are compositionally derived after first considering verb-meanings, projecting them lexically, and then the meanings of their complements, noun-phrases.

\subsubsection{Verbal meanings}

The main property of (eventive) verbs, besides combining with their complements (assumed here to be nounphrases), is their meaning inducing the above mentioned contextual modifier when projecting sentences.

We thus assume the following categorization of verbs. ${ }^{11}$

Intransitive verbs $v: S \backslash N P: \lambda x^{e} \lambda P^{(i(l t))} \lambda I^{i} \lambda M^{l} \exists J^{i} \exists L^{l} \cdot \mathbf{v}(x)(J, L) \wedge J \subseteq I \wedge L \subseteq M \wedge P(J, L)$.

Transitive verbs $v:(S \backslash N P) / N P: \lambda y^{e} \lambda x^{e} \lambda P^{(i(l))} \lambda I^{i} \lambda M^{l} \exists J^{i} \exists L^{l} \cdot \mathbf{v}(y, x)(J, L) \wedge J \subseteq I \wedge L \subseteq M \wedge P(J, L)$.

Thus, instead of the earlier version (19), we have

(24) $\llbracket \operatorname{kiss} \rrbracket=(S \backslash N P) / N P: \lambda y^{e} \lambda x^{e} \lambda P^{(i(l t))} \lambda I^{i} \lambda M^{l} \exists J^{i} \exists L^{l} \cdot \operatorname{kiss}(y, x)(J, L) \wedge J \subseteq I \wedge L \subseteq M \wedge P(J, L)$.

(Again, we note that the existential quantifiers here are place holders for a more constrained (but verbose) definition in terms of definites.)

In [26], there is a discussion of the origin of the structure of verbal meanings, seen there as having the temporal extent and quantifiers that bind it contributed by tense and aspect. The origin of the locative extent remains unspecified. In the remainder of the paper, we deal only with tensed sentences that start with a verb-contributed existential quantifier on event-extents. Here we briefly consider the more general case.

There are other verb categories which are partly specified by the prepositions involved and by verbal inflections (less completely in English than in, for example, Spanish or Russian), embodying universal quantification. A typical example is the following: ${ }^{12}$

\section{(25) Mary smiled whenever John kissed her.}

specified R rather vaguely as a temporal "point", whereas we specify it as a region of both time and space. Second, Reichenbach did not specify the implications for embedded clauses, or distinguish the relational use of $\mathrm{R}$ as either anaphoric or bound. We explicitly assign every tensed clause its own R-equivalent $I$ and $M$, and distinguish these elements from the contextual reference point "then/there". This last point is anticipated by [15], [27] and [28].

${ }^{10}$ We note in passing that, contrary to [7], the two event-extents are independent of each other, each being separately modified by an CPP. In [7] it is suggested that event-locations are functionally determined by event-times, an approach that disallows separate modifiability, and hence appears to be empirically incorrect.

${ }^{11}$ These particular verbs happen to have the most general slash-type. This is not always the case for verbal arguments.

${ }^{12}$ We note that where(ever) behaves as the spatial analog of when(ever) in this respect, as in John built a gazebo wherever Mary kissed him. 
In [25] and [24] this is accounted for by deriving its meaning from a more primitive, unquantified stem form (referred to as "undetermined" there), which in present terms can be presented as follows.

$$
\llbracket \operatorname{kiss} \rrbracket=\left(S_{s t e m} \backslash N P\right) / N P: \lambda x^{e} \lambda y^{e} \lambda J^{i} \lambda L^{l} \cdot \mathbf{k i s s}(x, y)(J, L)
$$

The verb inflection ${ }^{13}$ is responsible for both quantifier selection (i.e., determination), and binding by a quantifier the $\lambda$-bound temporal contextual argument, by a process discussed in section 3.1.7 under the name "finalization". This inflection could be viewed as a morpheme bearing one or more categories of the following general form (for the transitive case): ${ }^{14}$

$$
\left(\left(S_{\mathbf{A}, \mathbf{T}} \backslash N P\right) / N P\right) /\left(\left(S_{\text {stem }} \backslash N P\right) / N P\right): \lambda \mathcal{V}^{(e(e(i(l t))))} \cdot \delta_{\mathbf{q}_{\mathbf{A}}}(\mathcal{V})
$$

where $\mathbf{A}$ is an aspectual class such as event and $\mathbf{T}$ is a tense such as past, and $q_{\mathbf{A}}$ is the quantifier characterizing the aspectual class $\mathbf{A}$. Here $\delta_{\mathbf{q}}$ is an explicit semantic operation of determination that must take place as a lexical process, creating the quantified (including the $P^{(i(l t))}, I, M$ arguments) verb-meaning. This operator is given by (for a schematic quantifier $\mathbf{q}$, and a transitive verb),

$$
\delta_{\mathbf{q}}=\lambda \mathcal{F}^{(e(e(i(l t))))} \lambda x^{e} \lambda y^{e} \lambda P^{(i(l t))} \lambda I^{i} \lambda M^{l} . \mathbf{q}\left(\lambda J^{i} \lambda L^{l} \cdot \mathcal{F}(x, y)(J, L) \wedge J \subseteq I \wedge L \subseteq M, \lambda J^{i} \lambda L^{l} . P(J, L)\right)
$$

For example, the effect of past tense morphology upon the verb kiss is the eventive, existential quantifier case exclusively discussed in the previous sections:

$$
\begin{aligned}
\llbracket \text { kissed } \rrbracket= & \left(S_{\text {event,past }} \backslash N P\right) / N P: \delta_{\exists}\left(\lambda x^{e} \lambda y^{e} \lambda J^{i} \lambda L^{l} \cdot \operatorname{kiss}(x, y)(J, L)\right)= \\
& \lambda x^{e} \lambda y^{e} \lambda P^{(i(l t))} \lambda I^{i} \lambda M^{l} \exists J^{i} \exists L^{l} \cdot \operatorname{kiss}(x, y)(J, L) \wedge J \subseteq I \wedge L \subseteq M \wedge P(J, L)
\end{aligned}
$$

However, in English, unlike some other languages, past tense morphology (unlike the present tense) is ambiguous among a number of aspectual categories, including an iterative aspect, yielding a second category:

$$
\begin{aligned}
& \text { \kissed } \rrbracket=\left(S_{\text {iterative,past }} \backslash N P\right) / N P: \delta_{\exists}\left(\lambda x^{e} \lambda y^{e} \lambda J^{i} \lambda L^{l} \cdot \operatorname{kiss}(x, y)(J, L)\right)= \\
& \lambda x^{e} \lambda y^{e} \lambda P^{(i(l t))} \lambda I^{i} \lambda M^{l} \exists J^{i} \exists L^{l} \forall K^{i} \subseteq J \forall N^{l} \subseteq L \text {.kiss }(x, y)(K, N) \wedge J \subseteq I \wedge L \subseteq M \wedge P(J, L)
\end{aligned}
$$

Other determinations, such as habitual or futurate stative ([28]), are also required.

Thus, we follow [26] in attributing [25]'s operation of determination to the influence of tense and aspect. Since these are syntactically marked, prepositions and prepositional phrases are free to subcategorize for particular aspects.

Prepositional phrases like in every meeting $\backslash S_{S}$ are free to combine with either aspectual type (by convention, if an attribute is unrestricted as to value, we suppress the attribute-value feature entirely):

$$
\llbracket \operatorname{lin} \rrbracket=(S \backslash S) / N P: \ldots
$$

On the other hand the preposition whenever, exemplified in (25), demands iterative aspect in its first complement, and will combine only with sentences resulting from the latter aspectual version of kissed:

$$
\text { \whenever } \rrbracket=\left(S_{\text {iterative }} \backslash S_{\text {event }}\right) / S_{\text {iterative }}: \ldots
$$

Thus, in (25), the preposition whenever disambiguates the aspect of its complement.

Again, following [26] (in spirit if not to the letter), in the process of "finalizing" a sentence whose modification is complete, the syntactic tense marker additionally determines a binding for $I$ by conjoining a predication like $\operatorname{PAST}(I)$, FUTURE $(I)$, to the meaning derived above, as alluded to in our discussion of Finalization in section 3.1.7 below. We assume that a side-effect of such predication is to bind $I$ to a contextually available past reference time, related to Reichenbach's $R .^{15}$ It is an advantage of the present account that the purely syntactic propagation

\footnotetext{
${ }^{13}$ We refer here to what [26] calls the "semantic inflection", which may be distinct from its actual morphological realization; thus, past tense in English need not necessarily be realized by means of 'ed'.

${ }^{14}$ Naturally, one would want to schematize over intransitive, transitive, etc., verbal categories — we ignore such refinement for present purposes.

${ }^{15}$ Presumably, a similar spatial reference point must be available to bind the locative contextual variable $M$.
} 
of the tense feature from the lexical category of the tensed verb to the final sentence (over any number of CPP modifications), guarantees that it is the outermost $I$ that gets tense bound. This effect is achieved by purely local operations, escaping criticism by [26] of [25].

Thus, the logical form in (24) is only one among a number of other aspectual varieties induced by tensed morphology. A more detailed and complete account of these lexical processes in a categorial grammar framework over a full temporal ontology of the kind discussed in [28] remains a topic for further research. ${ }^{16}$

\subsubsection{Nominal and noun-phrase meanings}

The traditional meaning of nouns, of basic category $N$, is of type (et), predicates over entities, of the form $\lambda x^{e} \cdot \mathbf{n}(x)$. Here, as in [25], nouns acquire a relational meaning of type $(e(i(l t)))$, having contextual extents as arguments, and having their own extents located within the contextual ones (or functions thereof). Each entity $x$ of type $e$ that satisfies a nominal property will have associated with it by a model two extents, as follows.

- $\tau(x)$ : the temporal extent of $x$.

- $\sigma(x)$ : the locative extent of $x$.

The functions $\tau$ and $\sigma$ that map individuals onto temporal and spatial extents are discussed at length below in the context of temporal expressions like after every meeting and in a garden. In [25], this contextuality of nouns (and the relational meaning it induces) was attributed to them only within the scope of a (temporal) preposition. However, since individual descriptions such as the president of the United States also have temporal extents we do this for all common nouns. Note that this (double-)contextuality is needed both for generating correct noun-phrase meanings (as described below) and for supporting noun-modification by CPPs, as in examples (12) and (14), as described in Section 4.

Thus, the meaning of a common noun is given by

$$
\llbracket n \rrbracket=N: \lambda x^{e} \lambda I^{i} \lambda M^{l} \cdot \mathbf{n}(x) \wedge \tau(x) \subseteq I \wedge \sigma(x) \subseteq M
$$

For example,

$$
\llbracket \text { meeting } \rrbracket=N: \lambda x^{e} \lambda I^{i} \lambda M^{l} \text {.meeting }(x) \wedge \tau(x) \subseteq I \wedge \sigma(x) \subseteq M
$$

For a noun such as girl, when appearing in a non-temporal and non-locative context, we can assume (akin to a meaning postulate), that in every model

$$
\forall x^{e} \cdot \operatorname{girl}(x) \Rightarrow \tau(x)=\sigma(x)=\emptyset
$$

Thus, the two inclusions in the contextual extents are trivially satisfied and can be ommitted from logical forms (but see the discussion at the end of 3.1.3).

Further justification for having every noun doubly contextualized appears later on.

It is important here to note that, as common in all model-theoretic semantics, the extents assigned to nouns by models are arbitrary. Those extents can influence containment among elements in the noun's denotation. It is only world-knowledge (or other pragmatical considerations) that can exclude such "undesired" containments. Thus, by world knowlege, months contain weeks and not vice-versa; meetings take place within summers, but do not last over summers, etc. However, in arbitary models, such restrictions do not apply. We return to this point when discussing out-of-order CPP modification.

We now turn to noun-phrase meanings. The traditional meaning of Mary as a generalized quantifier is written as follows in the present notation:

$$
\llbracket \mathrm{Mary} \rrbracket=N P \uparrow: \lambda Q \cdot Q(\mathbf{m a r y})
$$

For example, consider the following minimal sentence:

\footnotetext{
${ }^{16}$ One kind of sentence, discussed in [26] (and attributed there to [11]) is that of "long dependencies", exemplified by

(26) Mary met John in New York before she claimed she would arrive.

There is a reading where the meeting time is before the (claimed) arrival time, and not before the claim itself. We have not yet considered intensional contexts of verbs requiring sentential complements. This too remains a question for further research.
} 


$$
\begin{gathered}
\llbracket \text { every } \rrbracket=\lambda P^{(e t)} \lambda Q^{(e \ldots t)} \lambda \ldots . \forall x^{e} . P(x) \Rightarrow Q(x \ldots) \\
\llbracket \text { a } / \text { some } \llbracket=\lambda P^{(e t)} \lambda Q^{(e \ldots t)} \lambda \ldots . . \exists x . P(x) \wedge Q(x \ldots) \\
\llbracket \text { the } \rrbracket=\lambda P^{(e t)} \lambda Q^{(e \ldots t)} \lambda \ldots . \exists ! x . P(x) \wedge \exists x . P(x) \wedge Q(x \ldots) \\
\llbracket \text { no } \rrbracket=\lambda P^{(e t)} \lambda Q^{(e . . t)} \lambda \ldots . . \forall x . P(x) \Rightarrow \neg Q(x \ldots)
\end{gathered}
$$

Figure 3: Standard determiner meanings

$$
\begin{aligned}
& \llbracket \text { every } \rrbracket=\lambda \mathcal{N}^{(e(i(l t)))} \lambda \mathcal{V}^{(e . . \vec{t})} \lambda \ldots \lambda P^{(i(l t))} \lambda I^{i} \lambda M^{l} . \forall x^{e} . \mathcal{N}(x)(I, M) \Rightarrow \mathcal{V}(x \ldots)(P, I, M) \\
& \llbracket \mathrm{a} / \text { some } \rrbracket=\lambda \mathcal{N} \mathcal{N}^{(e(i(l t)))} \lambda \mathcal{V}^{(e . . \vec{t})} \lambda \ldots \lambda P^{(i(l t))} \lambda I^{i} \lambda M^{l} . \exists x^{e} . \mathcal{N}(x)(I, M) \wedge \mathcal{V}(x \ldots)(P, I, M) \\
& \llbracket \text { the } \rrbracket=\lambda \mathcal{N} \mathcal{N}^{(e(i(l t)))} \lambda \mathcal{V}^{(e . . . t)} \lambda \ldots \lambda P^{(i(t))} \lambda I^{i} \lambda M^{l} . \exists ! x^{e} . \mathcal{N}(x)(I, M) \wedge \exists x^{e} . \mathcal{N}(x)(I, M) \wedge \mathcal{V}(x \ldots)(P, I, M) \\
& \llbracket \mathrm{no} \rrbracket=\lambda \mathcal{V}^{(e(i(l t)))} \lambda \mathcal{V}^{(e . . \vec{t})} \lambda \ldots \lambda P^{(i(l))} \lambda I^{i} \lambda M^{l} . \forall x^{e} . \mathcal{N}(x)(I, M) \Rightarrow \neg \mathcal{V}(x \ldots)(P, I, M)
\end{aligned}
$$

Figure 4: Revised determiner meanings

(27) Mary smiled.

The nominative instance of type-raising that is relevant to (27) is the following:

$$
\rrbracket \text { Mary } \rrbracket=S /(S \backslash N P): \lambda Q^{(e t)} \cdot Q(\text { mary })
$$

Such quantifier categories expect the semantic type of their argument to be a function $(e t)$. Figure 3 shows the usual determiner meanings inducing generalized quantifiers, whose standard syntactic category is $N P \uparrow / N$. The type $e \ldots t$ schematizes over the type of functions of any number of arguments whose first argument is of type $e$. The expression $\lambda \ldots$ schematizes over a sequence of abstractions over the remaining arguments, if any, and the expression $Q(x \ldots)$ schematizes over the application of $Q$ to all its arguments.

The traditional meanings of determiners and noun-phrases shown in Figure 3 must be adapted to apply to the new verbal categories of type $(e \vec{t})$, such as (24). Even though the standard meanings seem to be deeply rooted in semantic theories, they were proposed in a setting where a verb carried a simple predicative denotation of type (et). Now that verb denotations have been changed, a determiner expects a different complement denotation. Figure 4 shows the new denotations for determiners, inducing contextual modifiers when applied to noun-meanings and then to verb-meanings. Note that their syntactic category remains intact, as a function $N P \uparrow / N$ from nouns to type-raised $N P$. We refer to such meanings as temporal-locative generalized quantifiers (TLGQs).

For the sake of simplicity, we will treat proper nouns like $\llbracket \mathrm{Mary} \rrbracket$ as rigid designators (i.e., sameness of designation across times and locations), lexically raised to the level inducing an contextual Modifier after application to verbmeanings, as:

$$
\llbracket \text { Mary } \rrbracket=N P \uparrow: \lambda \mathcal{V} \lambda \ldots \lambda P^{(i(l t))} \lambda I^{i} \lambda M^{l} . \mathcal{V}(\mathbf{m}) \ldots(P, I, M)
$$

—where ... again stands for the arguments of the verb other than the first one. In the case of the nominative, there are no further arguments, so the instance relevant to (27) is as follows:

$$
\llbracket \text { Mary } \rrbracket=S /(S \backslash N P): \lambda \mathcal{V}^{(e \vec{t})} \lambda P^{(i(l t))} \lambda I^{i} \lambda M^{l} . \mathcal{V}(\mathbf{m})(P, I, M)
$$




\subsubsection{Sentential meanings}

The verb-meanings described above generate the required sentence-meanings as follows. (For brevity, we describe the intransitive case only.) First consider sentence (27), Mary smiled. From the above, we have

$$
\llbracket \text { smiled } \rrbracket=S \backslash N P: \lambda x^{e} \lambda P^{(i(l t))} \lambda I^{i} \lambda M^{l} \exists J^{i} \exists L^{l} . \text { smile }(x)(J, L) \wedge J \subseteq I \wedge L \subseteq M \wedge P(J, L)
$$

$\llbracket$ Mary smiled $\rrbracket=\llbracket$ Mary $\llbracket(\rrbracket$ smiled $\rrbracket)=$

$S: \lambda \mathcal{V}^{(e \vec{e})} \lambda P^{(i(l t))} \lambda I^{i} \lambda M^{l} . \mathcal{V}(\mathbf{m a r y})(P, I, M)$

$\left(\lambda x^{e} \lambda P^{(i(l t))} \lambda I^{i} \lambda M^{l} \exists J^{i} \exists L^{l}\right.$.smile $\left.(x)(J, L) \wedge J \subseteq I \wedge L \subseteq M \wedge P(J, L)\right)=$

$\lambda P^{(i(l t))} \lambda I^{i} \lambda M^{l} \exists J^{i} \exists L^{l}$.smile(mary) $(J, L) \wedge J \subseteq I \wedge L \subseteq M \wedge P(J, L)$

as expected.

Consider a subject-quantified sentence like the following:

(28) Every girl smiled.

The noun-phrase has the following revised interpretation:

$$
\text { \every girl] }=S /(S \backslash N P): \lambda \mathcal{V}^{(e \vec{t})} \lambda P^{(i(l t))} \lambda I^{i} \lambda M^{l} \forall x^{e} \cdot \operatorname{girl}(x) \wedge \tau(x) \subseteq I \wedge \sigma(x) \subseteq M \Rightarrow \mathcal{V}(x)(P, I, M)
$$

The meaning of (28) is therefore the following:

[Every girl smiled $\rrbracket=\llbracket$ every girl $\rrbracket(\rrbracket$ smiled $\rrbracket)=$

$$
\begin{aligned}
& S: \lambda P^{(i(l t))} \lambda I^{i} \lambda M^{l} \forall x^{e} \text {.girl }(x) \wedge \tau(x) \subseteq I \wedge \sigma(x) \subseteq M \\
& \Rightarrow \exists J^{i} \exists L^{l} \text {.smile }(x)(J, L) \wedge J \subseteq I \wedge L \subseteq M \wedge P(J, L)
\end{aligned}
$$

Since girl can be viewed here as a non-temporal, non-locative noun, based on the assumption on $\tau, \sigma$ for such nouns this reduces to $S: \lambda P^{(i(l t))} \lambda I^{i} \lambda M^{l} \forall x^{e}$. $\operatorname{girl}(x) \Rightarrow \exists J^{i} \exists L^{l}$.smile $(x)(J, L) \wedge J \subseteq I \wedge L \subseteq M \wedge P(J, L)$.

It is important here that there is no direct relationship between $\tau(x)$ and $I$. Thus, for (a variant of) Enc's [8] example

(29) Every fugitive rejoiced (in a garden party, on the anniversary of her escape)

the restriction is that $\tau(x) \subseteq I$, i.e., there is/was a time within the contextual time $I$, at which $x$ is/was a fugitive; such a time is not related to (and certainly need not be equal to) the event-time of rejoicing, $J$. Thus, the correct prediction is obtained. Note that this issue is quite orthogonal to our main interest, namely, CPP-modification. Therefore, we henceforth assume that nouns in the main clause bear atemporal meanings (like girl above), satisfying $\tau(x)=\sigma(x)=\emptyset$, to simplify the presentation.

We discuss a further process of finalization, whereby sentential meaning reduces further to a specific proposition, in section 3.1.7.

\subsubsection{Temporal and locative prepositions}

The preposition meanings given below have the following characteristics:

- They assume that adjuncts semantically compose their meanings with the sentential meanings that they modify.

- They contribute ${ }^{17}$ an context-shift (either temporal or locative) to the compositional meaning construction. More precisely, every preposition $p$ has associated with it a shift-function $F_{p}$, of a contextual-argument and

\footnotetext{
${ }^{17}$ Of course, the same prepositions may have additional meanings. For example, as noted by a referee, in the sentence

(30) In most of John's classes, he fails exactly three students

the preposition 'in' does not shift any extent of the failing event(s). We are concerned in this paper only with the context-shift meaning of temporal/locative prepositions.
} 
an event-extent. For a temporal $p$, the function is $F_{p}\left(I^{i}, J^{i}\right)$, while for a locative $p$ the function is $F_{p}\left(M^{l}, L^{l}\right)$. These functions carry the actual lexical meaning of the preposition ${ }^{18}$.

- They omit any detailed specification of those lexical meanings. To do so would require a richer ontology and mathematical treatment. Some are easy. For example, $F_{\text {after }}(I, J)$ yields the subinterval from the end of $J$ to the end of $I$, while $F_{\text {before }}(I, J)$ yields the subinterval from the beginning of $I$ to the beginning of $J$ (where $J \subseteq I$ for both cases). We refer to [25] for more details, and for other interpretations, e.g., "just before". We will not even try to describe $F_{\text {behind }}(M, L)$ in words. See, for example, [31] for the finer locative relations' specification via vectors.

Some prepositions, like during, in, ... have a trivial shift of the form $F_{p}(I, J)=J$, and could have been simply omitted. We retain them in logical forms for the sake of a transparent, uniform treatment.

Another major concern, the importance of which will become apparent when "long" cascades of CPPs are considered, is that TPP-meanings and LPP-meanings should be mutually composable (in both directions). This means, that viewing each kind of CPP as modifying one context only will not work - it will block composability. We need each CPP-meaning to relate both extents, leaving one intact and modifying the range of quantification for the other. Another argument for the double-contextualization of all temporal/locative nouns is presented when noun-attachment of CPPs is discussed.

All this leads to the following categorization of the temporal preposition during (and the like ${ }^{19}$ - deferring treatment of TPs like before, after, which involve a non-trivial time-shift, to a later stage), and the locative preposition in. For post-sentential CPP, we have the following types: ${ }^{20}$

(31) $\llbracket$ during/in $\rrbracket=(S \backslash S) / \Delta P: \lambda x^{e} \lambda \overrightarrow{S^{t}} \lambda P^{(i(l t))} \lambda I^{i} \lambda M^{l} . S\left(P, F_{\text {during } / \text { int }_{t}}(I, \tau(x)), M\right)$

(32) $\llbracket \operatorname{in} \rrbracket=(S \backslash S) \iota_{\diamond} N P: \lambda x^{e} \lambda \overrightarrow{S^{t}} \lambda P^{(i(l t))} \lambda I^{i} \lambda M^{l} . S\left(P, I, F_{i n_{l}}(M, \sigma(x))\right)$

Note that temporal prepositions shift only the temporal context, while locative prepositions shift only the locative context.

The above leads to the following kind of TPP-meaning:

(33) $\llbracket$ during/in every meeting $\rrbracket=S \backslash S$ : \every meeting $\llbracket(\llbracket$ during/in $\rrbracket)=$

$$
\begin{aligned}
& \lambda \overrightarrow{\mathcal{S}^{t}} \lambda P^{(i(l t))} \lambda I^{i} \lambda M^{l} \forall x \text {. meeting }(x) \wedge \tau(x) \subseteq I \wedge \sigma(x) \subseteq M \\
& \Rightarrow \mathcal{S}\left(P, F_{\text {during } / \text { in }_{t}}(I, \tau(x)), M\right)
\end{aligned}
$$

Similarly for LPP:

[in a garden $]=S \backslash S:$ la garden $\rrbracket(\rrbracket$ in $\rrbracket)=$

$$
\lambda \mathcal{S}^{\vec{t}} \lambda P^{(i(l t))} \lambda I^{i} \lambda M^{l} \exists x . \operatorname{garden}(x) \wedge \tau(x) \subseteq I \wedge \sigma(x) \subseteq M \wedge \mathcal{S}\left(P, I, F_{i n_{l}}(M, \sigma(x))\right)
$$

Note that the NPs are raised here (over the preposition type) to a higher type, for example

[every meeting $\llbracket=N P \uparrow=(S \backslash S) \backslash((S \backslash S) / N P)$ :

$\lambda \mathcal{F}^{(e(\overrightarrow{t t}))} \lambda S^{\vec{t}} \lambda P^{(i(l t))} \lambda I^{i} \lambda M^{l} \forall x^{e}$.meeting $(x) \wedge \tau(x) \subseteq I \wedge \sigma(x) \subseteq M \Rightarrow \mathcal{F}(x)(S, P, I, M)$

\footnotetext{
${ }^{18}$ But see the discussion on veridicality in 5.0.1

${ }^{19}$ Some prepositions, for example in, have both a temporal and locative meaning. For ease of reading, we denote them in logical forms as $i n_{t}$ and $i n_{l}$, respectively.

${ }^{20}$ The "associative" modality $\diamond$ is used in the IP categories to permit preposition stranding to occur via harmonic composition. On other assumptions the more restrictive modality $\star$ would be appropriate. The unrestricted slash on the modifier slash is required to permit "Heavy NP shifted" sentences like Harry saw in the garden a beautiful bed of roses via backward crossed composition ([27]). We defer discussion of these topics to a later paper.
} 


\subsubsection{CPP modification}

Finally, we reach the stage of sentence modification via CPP. The CPP is syntactically applied (as dictated by its category) to the sentence. However, the CPP-meaning has the effect of lexically function-composing the component contextual modifiers. Bound variables are renamed for readability.

TPP-modification: We compute the meaning of (2).

\Mary kissed John in every meeting $\rrbracket=$ \in every meeting $\llbracket(\rrbracket$ Mary kissed John $\rrbracket)$

$S: \lambda P^{(i(l t))} \lambda I^{i} \lambda M^{l} \forall x$.meeting $(x) \wedge \tau(x) \subseteq I \wedge \sigma(x) \subseteq M \Rightarrow \exists J_{0} \exists L_{0}$. kiss(mary,john) $\left(J_{0}, L_{0}\right)$

$\wedge J_{0} \subseteq F_{\text {in }}(I, \tau(x)) \wedge L_{0} \subseteq M \wedge P\left(J_{0}, L_{0}\right)$.

Paraphrasing, this means that (as expected) for every pair of event-extents within the context, which are extents of a meeting-event, there is a subinterval of the meeting temporal extent, which together with the contextual locative extent form the event-extents of a kissing event (of John by Mary).

LPP-modification: Recomputing the meaning of (4), we get

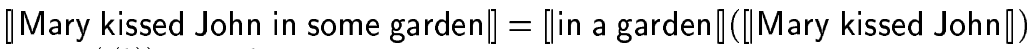

$S: \lambda P^{(i(t))} \lambda I^{i} \lambda M^{l} \exists x$.garden $(x) \wedge \tau(x) \subseteq I \wedge \sigma(x) \subseteq M \wedge \exists J_{0} \exists L_{0}$.kiss(mary,john $)\left(J_{0}, L_{0}\right)$

$$
\wedge J_{0} \subseteq I \wedge L_{0} \subseteq F_{\text {inl }}(M, \sigma(x)) \wedge P\left(J_{0}, L_{0}\right) .
$$

Similarly, the location of the kissing is included within that of the garden, while the kissing-time is only restricted by the temporal context.

The prepositions considered so far were those that only restrict the basic event-extents by inclusion in the respective event-extents of the modifier - that is, where $F_{\text {during }}(I, \tau(x))=\tau(x), F_{\text {inl }}(M, \sigma(x))=\sigma(x)$, and so on. As mentioned before, another major class of prepositions induce more complicated relations between the event-extents.

We get the following lexical categorizations.

Пafter $\rrbracket=(S \backslash S) /_{0} N P:=\lambda x^{e} \lambda S^{\vec{t}} \lambda P^{(i(l t))} \lambda I^{i} \lambda M^{l} \cdot S\left(P, F_{\text {after }}(I, \tau(x)), M\right)$

(Another variant of before, after, taking sentential complements, is discussed in Section 5). Similarly for behind, with $F_{\text {behind }}$ replacing $F_{\text {after }}$ :

[behind $\rrbracket=\left.(S \backslash S)\right|_{0} N P: \lambda x^{e} \lambda \overrightarrow{S^{l}} \lambda P^{(i(l t))} \lambda I^{i} \lambda M^{l} . S\left(P, I, F_{\text {behind }}(M, \sigma(x))\right)$

Consider now the following examples.

(34) Mary kissed John after some meeting.

(35) Mary kissed John behind every tree.

By similar derivations as before we obtain

\Mary kissed John after some meeting $\rrbracket=\rrbracket$ after some meeting $\llbracket(\rrbracket$ Mary kissed John $\rrbracket)=$ $S: \lambda P^{(i(l t))} \lambda I^{i} \lambda M^{l} \exists x$.meeting $(x) \wedge \tau(x) \subseteq I \wedge \sigma(x) \subseteq M$ $\wedge \exists J_{0} \exists L_{0}$. kiss $($ mary, john $)\left(J_{0}, L_{0}\right) \wedge J_{0} \subseteq F_{\text {after }}(I, \tau(x)) \wedge L_{0} \subseteq M \wedge P\left(J_{0}, L_{0}\right)$.

Here, the kissing-event is shifted to a time subsequent to the meeting-time, and again the kissing-location is unrelated to the meeting-location, restricted only by the locative context. Similarly,

\Mary kissed John behind every tree $\rrbracket=\llbracket$ behind every tree $\llbracket(\llbracket$ Mary kissed John $\rrbracket)=$

$S: \lambda P^{(i(l t))} \lambda I^{i} \lambda M^{l} \forall x$.tree $(x) \wedge \tau(x) \subseteq I \wedge \sigma(x) \subseteq M$

$$
\Rightarrow \exists J_{0} \exists L_{0} . \mathbf{k i s s}(\text { mary,john })\left(J_{0}, L_{0}\right) \wedge J_{0} \subseteq I \wedge L_{0} \subseteq F_{\text {behind }}(M, \sigma(x)) \wedge P\left(J_{0}, L_{0}\right) .
$$


We have been assuming, for ease of presentation, that CPPs are sentential modifiers. However, it is well-known that on a finer analysys, CPPs should be VP-modifiers, to allow handling, for example, Verb-Phrase Ellipsis (VPE), as in

(36) Mary kissed John in every meeting, and Sue did too.

We sketch here how such a refinement can be carried out.

First, the category of prepositions is slightly changed, so as to render a CPP a predicate modifier. Abbreviating $S \backslash N P$ to $V P$, we put

$$
\begin{aligned}
& \llbracket \operatorname{in} \rrbracket=\left.(V P \backslash V P)\right|_{\diamond} N P: \lambda x^{e} \lambda \mathcal{V} \mathcal{P}^{(\overrightarrow{e t})} \lambda P^{(i(l t))} \lambda I^{i} \lambda M^{l} \lambda z^{e} . \mathcal{V} \mathcal{P}(z)\left(P, F_{\text {during } / \text { int }_{t}}(I, \tau(x)), M\right) \\
& \text { [in every meeting } \mid=V P \backslash V P: \lambda \mathcal{V} \mathcal{P}^{(\overrightarrow{e t})} \lambda P^{(i(l t))} \lambda I^{i} \lambda M^{l} \lambda z^{e} \forall x \text {.meeting }(x) \wedge \tau(x) \subseteq I \wedge \sigma(x) \subseteq M \\
& \Rightarrow \mathcal{V} \mathcal{P}(z)\left(P, F_{\text {during } / \text { int }_{t}}(I, \tau(x)), M\right)
\end{aligned}
$$

which results in

\kissed John in every meeting $\rrbracket=$

$V P: \lambda z^{e} \lambda P^{(i(l t))} \lambda I^{i} \lambda M^{l} \forall x$.meeting $(x) \wedge \tau(x) \subseteq I \wedge \sigma(x) \subseteq M \Rightarrow \exists J_{0} \exists L_{0} \cdot \mathbf{k i s s}(z$, john $)\left(J_{0}, L_{0}\right)$

$$
\wedge J_{0} \subseteq F_{\text {in }}(I, \tau(x)) \wedge L_{O} \subseteq M \wedge P\left(J_{0}, L_{0}\right) .
$$

This $V P$ is now available as an antecedent for $V P E$ by whichever method of Categorial Grammar used in general for $V P E$. Again, for keeping the presentation simpler, we retain viewing CPPs a sentential modifiers.

\subsubsection{Cascaded CPP-modification}

Suppose we now want to calculate the meaning of (3). Then, by

[Mary kissed John in every meeting during some summer $]=$ \during some summer $(\llbracket$ Mary kissed John in every meeting $\llbracket)$ $=\rrbracket$ during some summer $\rrbracket(\rrbracket$ in every meeting $\llbracket(\rrbracket$ Mary kissed John $\rrbracket))$

$=S: \lambda P^{(i(l t))} \lambda I^{i} \lambda M^{l} \exists y^{e} \cdot \operatorname{summer}(y) \wedge \tau(y) \subseteq I \wedge \sigma(y) \subseteq M$

$$
\begin{aligned}
& \wedge\left[\forall x^{e} \text {. meeting }(x) \wedge \tau(x) \subseteq F_{\text {during }}(I, \tau(y)) \wedge \sigma(x) \subseteq M\right. \\
& \left.\left.\Rightarrow \exists J_{0}^{i} \exists L_{0}^{l} \text {.kiss(mary,john }\right)\left(J_{0}, L_{0}\right) \wedge J_{0} \subseteq F_{i n_{t}}(\tau(y), \tau(x)) \wedge L_{0} \subseteq M \wedge P\left(J_{0}, L_{0}\right)\right] .
\end{aligned}
$$

This reduction establishes the required cascaded meaning, which can be paraphrased as follows: there are a temporal interval and a spatial region (within the contextual parameters) which are a summer time and location, and every subinterval of the temporal extent which is a meeting-time, itself contains a subinterval which, together withsome subregion of the locative context, are an event-time and an event-location of a kissing of John by Mary.

Note the scope relations of the quantifiers: The last-attached CPP introduces the quantifier with the highest scope. This has a certain effect when the cascading includes time-shifting prepositions. Consider the following example.

(37) Mary kissed John before a meeting on some morning.

For the cascaded attachment, employing the same method as before, we get

$$
\begin{aligned}
&=S: \lambda P^{(i(l t))} \lambda I^{i} \lambda M^{l} \exists y^{e} \text {.morning }(y) \wedge \tau(y) \subseteq I \wedge \sigma(y) \subseteq M \\
& \wedge\left[\exists x . \text { meeting }(x) \wedge \tau(x) \subseteq F_{\text {int }}(I, \tau(y)) \wedge \sigma(x) \subseteq M\right. \\
&\left.\wedge \exists J_{0}^{i} \exists L_{0}^{l} \cdot \mathbf{k i s s}(\mathbf{m a r y}, \text { john })\left(J_{0}, L_{0}\right) \wedge J_{0} \subseteq F_{\text {before }}(\tau(y), \tau(x)) \wedge L_{0} \subseteq M \wedge P\left(J_{0}, L_{0}\right)\right] .
\end{aligned}
$$

Under this reading, the kissing has to occur between the beginning of the morning and the beginning of the meeting. We refer to this as the "short" reading. This sentence has also a "long" reading, in which the kissing may occur between the beginning of the contextual interval and the beginning of the meeting (not necessarily in the morning). We return to this reading, obtained via a noun-attachment of on some morning, in Section 4. A similar ambiguity, but related to three-dimensional regions, obtains for a sentence like 
(38) Mary kissed John outside a garden in some city.

So far, interpretations for constructions of the general form sentence $C P P_{1} C P P_{2}$ have been derived by application, schematizable as follows:

$$
\frac{\frac{S: \mathbf{s} S \backslash S: \mathbf{C P P}_{\mathbf{1}}}{S: \mathbf{C P P}_{\mathbf{1}}(\mathbf{s})}<}{S: \mathbf{C P P}_{\mathbf{2}}\left(\mathbf{C P P}_{\mathbf{1}}(\mathbf{s})\right)}<
$$

An alternative derivation using the backward harmonic composition rule $<\mathbf{B}$ from Figure 1 is of course guaranteed to yield the same interpretation:

$$
\text { (39) } \begin{aligned}
S: \mathbf{s} S \backslash S: \mathbf{C P P}_{\mathbf{1}} \quad S \backslash S: \mathbf{C P P}_{\mathbf{2}} \\
\frac{S \backslash S: \lambda x \cdot \mathbf{C P P}_{\mathbf{2}}\left(\mathbf{C P P}_{\mathbf{1}}(x)\right)}{S: \mathbf{C P P}_{\mathbf{2}}\left(\mathbf{C P P}_{\mathbf{1}}(\mathbf{s})\right)}<
\end{aligned}
$$

That is, for the running example, we would get the following category for the fragment during every meeting in some summer:

$$
\begin{aligned}
S \backslash S: \lambda \vec{S}^{\vec{t}} \lambda P^{(i(l t))} \lambda I^{i} \lambda M^{l} \exists y^{e} \cdot \operatorname{summer}(y) \wedge \tau(y) \subseteq I \wedge \sigma(y) \subseteq M & \\
& \wedge \\
& {\left[\forall x^{e} \text {.meeting }(x) \wedge \tau(x) \subseteq F_{i n_{t}}(I, \tau(y)) \wedge \sigma(x) \subseteq M \Rightarrow S\left(P, F_{i n_{t}}(\tau(y), \tau(x)), M\right)\right] }
\end{aligned}
$$

This is a TLGQ or sentential modifier category, which when applied to the interpretation of Mary kissed John will yield the same interpretation as before. Generalizing to arbitrary cascades, we can view their meaning as resulting from first composing all CPP-meanings (still in their surface order), an observation that will be important in section 6 , where we discuss the fact that all such cascades can (under the assumption that all "like types" can coordinate) undergo "non-constituent" coordination, as in sentences like the following:

(40) Mary kissed John [during every meeting in some summer $]_{S \backslash S}$ and [during every reception in some winter $]_{S \backslash S \text {. }}$

\subsubsection{Finalization of sentential meaning and entailment}

An alert reader will have noticed that the meanings we attribute to (affirmative) sentences do not conform to the traditional view of them as expressing propositions and taking part in entailment. To reconcile our approach with the traditional view, we have to "finalize" (as in [25]) the sentential meanings with a "once-only" semantic operation, taking place once it is determined that the sentence is neither a complement of another preposition, nor is it further modified by another CPP. There are two points to take care of.

- We have to "get rid" of $\lambda P^{(i(t))}$ and the conjunct $P(J, L)$ in the sentential meaning. We do it by viewing the sentence as a complement of a "fictitious", vacuous, preposition, binding $P$ to the constant predicate $P_{0}=$ $\lambda J^{i} \lambda L^{l}$.true, holding for every pair of extents. The conjunct true can be safely removed from the meaning expression. As we shall see in the sequel (when considering sentential complements of prepositions), this part of the finalization need not take place at the very end of a derivation. Rather, it takes place when the $P$ argument is not needed anymore, i.e., the sentence will no longer need to capture extents under its scope. The very end is just one such occasion. Let us refer to this binding of $P$ to the trivial contextual relation as "semi-finalization".

- We have to decide how to treat the binding of the contextual-extents $I, M$. There seem to be two reasonable ways to proceed. 
1. Bind $I$ to the whole ${ }^{21}$ real line, and $M$ to the whole space. This results in a meaning resembling traditional representations of eventive sentential meanings, as existentially quantified over event-extents.

2. Leave $I, M$ as free variables, getting values as usual from an assignment $g$, which participates in the satisfaction and entailment relations.

The latter seems the better option, as it opens the way to incorporating sentential meaning into discourse, that can determine the assignments to $I$ and $M$ in much the same way as the compositional account, albeit via anaphoric reference. This issue touches issues of (formal) pragmatics, and we will not elaborate on it any further. However, we note that something of this kind is clearly necessary, if we are ever to correctly interpret the "pronominal" effect of tense in utterances like the following, when uttered in a car driving along the highway (from [23]):

(41) I left the oven on.

\subsection{Preposed cascades}

Consider now (6), repeated below.

(42) In every meeting, Mary kissed John.

In order to handle this surface order, the CPP has to have the following rightward-looking category, identical in meaning to the leftward-looking version:

(43) $\llbracket$ In every meeting $\rrbracket=S / S_{*}$

Unlike the backward IP category, the forward one must be modally restricted using the "application only" modality, in order to block overgeneralization of word order and the parasitic gap construction discussed in [27]. We defer detailed discussion of this restriction to a later occasion.

\subsection{Out-of-order cascading}

Consider the following sentence, permuting the order of CPPs in (3).

(44) Mary kissed John during some summer in every meeting.

By employing the technique delineated in Subsection 3.1, we obtain the following meaning, which says that every meeting-time contains a summer-time, which contains a kissing event-time. This meaning might be ruled out due to world knowledge, where meetings do not span over summers.

$$
\begin{aligned}
S: \lambda P^{(i(l t))} \lambda I^{i} \lambda M^{l} M^{l} & \forall y^{e} \text {.meeting }(y) \wedge \tau(y) \subseteq I \wedge \sigma(y) \subseteq M \\
& \Rightarrow\left[\exists x^{e} \text {.summer }(x) \wedge \tau(x) \subseteq F_{\text {int }}(I, \tau(y)) \wedge \sigma(x) \subseteq M \wedge\right. \\
& \left.\exists J_{0}^{i} \exists L_{0}^{l} . \mathbf{k i s s}(\text { mary, john })\left(J_{0}, L_{0}\right) \wedge J_{0} \subseteq F_{\text {during }}(\tau(y), \tau(x)) \wedge L_{0} \subseteq M \wedge P\left(J_{0}, L_{0}\right)\right] .
\end{aligned}
$$

However, (44) also carries a more reasonable meaning equivalent to that of (3), namely

$$
\begin{aligned}
S: \lambda P^{(i(l t))} \lambda I^{i} \lambda M^{l} \exists x^{e} \text {.summer }(x) \wedge \tau(x) \subseteq I \wedge \sigma(x) \subseteq M & \\
& \wedge\left[\forall y^{e} \text {.meeting }(y) \wedge \tau(y) \subseteq F_{\text {during }}(I, \tau(x)) \wedge \sigma(y) \subseteq M \Rightarrow\right. \\
& \left.\left.\exists J_{0}^{i} \exists L_{0}^{l} \text {.kiss(mary,john }\right)\left(J_{0}, L_{0}\right) \wedge J_{0} \subseteq F_{\text {int }}(\tau(x), \tau(y)) \wedge L_{0} \subseteq M \wedge P\left(J_{0}, L_{0}\right)\right] .
\end{aligned}
$$

To get this reading, during some summer must get scope over in every meeting. By assumption this must be accomplished directly by the combinatorics of syntactic derivation.

\footnotetext{
${ }^{21}$ Or its part compatible with the sentence tense, in case tense is added to the analysis following [26]; see discussion in the section 3.1.1.
} 
This reading can be analysed in semantic terms as the application of an "out of order" composition of the two modifier meanings to the core proposition. Since CPPs also have the "rightward-looking" category that was introduced to handle preposed CPPs, as in (42), there is a temptation at this point to toy with the idea of deriving the desired reading syntactically via the rule of forward crossed composition, $>B_{\times}$, as follows:

$$
\begin{gathered}
S: \mathbf{s} S / S: \mathbf{C P P}_{\mathbf{1}} \quad S \backslash S: \mathbf{C P P}_{\mathbf{2}} \\
\frac{S \backslash S: \lambda x \cdot \mathbf{C P P}_{\mathbf{1}}\left(\mathbf{C P P}_{\mathbf{2}}(x)\right)}{\left.S: \mathbf{B}_{\times *}\right)} \\
\hline \mathbf{C P P}_{\mathbf{1}}\left(\mathbf{C P P}_{\mathbf{2}}(\mathbf{s})\right)
\end{gathered}
$$

However, this temptation has to be resisted. First, the above derivation is currently ruled out by the modality of the forward modifier preposition category. Relaxing the modality immediately lets in the overgenerations referred to in section 3.2 which motivated the original restriction.

More importantly, when we come to discuss noun modifier CPPs in section 4, we will find that exactly the same crossing readings are available for NPs like the subject of the sentence Some meeting last summer in every conference was cancelled. Yet noun modifiers have no forward-modifying category, as is evident from the ill-formedness of the following:

(45) *an in some summer meeting

A further reason to eschew crossed composition of modifiers is that it compromises the Church-Rosser property of the calculus: derivations that are equivalent when normalized no longer deliver identical interpretations.

We must therefore exercise the only remaining degree of freedom that CCG allows, and achieve the same effect of "out of order" composition via a further lexical entry for IPs. The category of CPPs themselves can be schematized as follows: ${ }^{22}$

(46) $\llbracket C P P \rrbracket=\left.(S \backslash S)\right|_{\star}(S \backslash S): \lambda \mathcal{A}^{(\overrightarrow{t r t})} \lambda \overrightarrow{S^{t}} \cdot \mathbf{C P P}(\mathcal{A}(S))$

The corresponding temporal and locative categories for in/during as follows (cf. (31) and (32)

(47) [during $/ \mathrm{in}_{\mathrm{t}} \rrbracket=\left.\left(\left.(S \backslash S)\right|_{\star}(S \backslash S)\right)\right|_{\star} N P: \lambda x^{e} \lambda \mathcal{A}^{(\overrightarrow{t \overrightarrow{t t}})} \lambda \overrightarrow{\mathcal{S}^{t}} \lambda P^{(i(l t))} \lambda I^{i} \lambda M^{l} . \mathcal{A}(\mathcal{S})\left(P, F_{\text {during } / \text { int }_{t}}(I, \tau(x)), M\right)$

(48) $\llbracket \operatorname{in} \|=\left.\left(\left.(S \backslash S)\right|_{\star}(S \backslash S)\right)\right|_{\star} N P: \lambda x^{e} \lambda \mathcal{A}^{(\overrightarrow{t t})} \lambda \mathcal{S}^{\vec{t}} \lambda P^{(i(l t))} \lambda I^{i} \lambda M^{l} . \mathcal{A}(S)\left(P, I, F_{i n_{l}}(M, \sigma(x))\right)$

These IP categories then reduce by the application of a raised NP to them, as in (33), to give "out-of-order composing" TPP categories like the following, which is an instance of schema (46):

(49) $\llbracket$ in every meeting $\rrbracket=\llbracket$ every meeting $\llbracket(\llbracket$ in $\rrbracket)=$

$$
\left(\left.(S \backslash S)\right|_{\star}(S \backslash S)\right): \lambda \mathcal{A}^{(\vec{t})} \lambda S^{\vec{t}} \lambda P^{(i(l t))} \lambda I^{i} \lambda M^{l} \forall x^{e} \cdot \mathcal{A}(S)\left(P, F_{i n}(I, \tau(x)), M\right)
$$

For example, in (44) Mary kissed John in some summer during every meeting, this category yields the following "cross-composed" meaning for the fragment in some summer during every meeting:

$$
\begin{aligned}
=\lambda \overrightarrow{S^{t} \lambda} P^{(i(l t))} \lambda I^{i} \lambda M^{l} \exists x^{e} \text {.summer }(x) \wedge \tau(x) \subseteq I \wedge \sigma(x) \subseteq M & \\
& \hat{}\left(\forall y^{e} \text {.meeting }(y) \wedge \tau(y) \subseteq F_{\text {int }_{t}}(I, \tau(x)) \wedge \sigma(y) \subseteq M \Rightarrow S\left(P, F_{i n_{t}}(\tau(x), \tau(y)), M\right)\right]
\end{aligned}
$$

CPPs with the new category can also cross-compose CPPs of different kinds, temporal and locative.

Since both combining IIPs may themselves have been derived by composition—for example as in (39) - the scopepermuting category (46) allows many alternative readings. Thus for a cascade of $n \geq 2$ CPPs, we can, by judicious choice between scope-inverting and -non-inverting CPP categories, and between sequences of composition versus

\footnotetext{
${ }^{22}$ The $\star$ modalities are simply to play safe in the absence of any reason to generalize.
} 
application, generate permuted interpretations of the CPPs, inducing quantifier scopes not necessarily in the surface order. (This observation also is relevant to the discussion of coordination below, since coordinates like (40) can now carry such permuted meanings.)

The same permutability inheres to preposed CPP cascades for the scope-inverting category.

However, for $n>3$, not every permutation of $(1, \ldots, n)$ is generable. For example, for four CPPs, the following permutation is not generable, forcing the prediction that the corresponding reading is not available.

$$
\llbracket C P P_{3} \rrbracket \circ \rrbracket C P P_{1} \rrbracket \circ \llbracket C P P_{4} \| \circ \llbracket C P P_{2} \rrbracket
$$

In a generable permutation, there has to exist a pair of consecutive indices $\langle i,(i+1)\rangle$ or $\langle(i+1), i\rangle$; such a pair is the result of the very first reduction. The above permutation $(3,1,4,2)$ does not satisfy this condition and is therefore non-generable.

Unfortunately, it is hard to verify this prediction of a limit on IIP scope scrambling, since it only applies to cascades of length greater than 3. It is hard to interpret long CPP-cascades, and even harder to form such cascades that are neutral with regards to world knowledge. As an approximation to such an idealization, consider

(50) Mary kissed John (1) in a conference (2) on a weekend (3) during every meeting (4) before every vacation.

Because the previously-mentioned permutation is not generable, the following paraphrase is a reading predicted not to exist for (50): (within the contextual interval) there is a weekend s.t. for every vacation within it, there is a conference within the vacation, s.t. for every meeting within that conference Mary kissed John. On the other hand, since $(2,1,4,3)$ is a generable permutation of $(1,2,3,4)$, the following paraphrase is predicted to be an available reading: (within the contextual interval) for every meeting and every vacation within it, there is a conference within that vacation and a weekend within the conference during which Mary kissed John.

When there is both a pre-sentential CPP-cascade and a post-sentential one, our account predicts that each will permute independently. No reading will be available where the two sets of scopes intercalate: either the whole preposed cascade (possibly internally permuted) has scope over the post-posed cascade (also possibly internally permuted), or vice-versa.

\section{Noun modification by CPPs}

Consider the following variant of (12).

(51) Mary kissed John [ ${ }_{C P P}$ in every meeting][CPPOn a Monday].

Under the previous analysis, we get the meaning paraphrased as: there exists a Monday s.t. in every meeting on that Monday a kissing event took place. However, the more natural reading is paraphrasable as: during every meeting on any Monday, Mary kissed John. Thus, the universal quantifier on meeting times and locations outscopes the existential quantifier on Mondays. This interpretation seems to reflect a noun-modifying bracketing, as follows:

(52) Mary kissed John [CPPin every $\left[{ }_{n}\right.$ meeting on a Monday $]$.

Similar considerations apply to the following sentence:

(53) Mary kissed John [CPP in every meeting][CPPin a garden].

Again, under the previous analysis, we get the reading: there exists a garden, such that in every meeting in it, Mary kissed John. Once again, the more natural reading is: Mary kissed John in every meeting in any garden; the universal quantifier on meeting times and locations again outscopes the existential quantifier on garden locations, reflecting the folowing bracketing: 
(54) Mary kissed John [

One might expect it to be possible to separate the temporal and locative nouns, having each kind induce its own $\mathrm{CPP}^{23}$ We mentioned already that for mutual composability of the induced CPP-meaning (needed for out-oforder evaluation), all nouns have to be doubly-contextualized. Below we present another argument for doublecontextuality of all nouns.

The argument comes from the existence of nouns that can be both temporally and locatively modified by CPPattachment to them. Thus we have both

(55) meeting in a garden

(56) meeting in a summer

and even

(57) meeting in a summer in a garden

It may even be the case that the same noun is used both for temporal and locative modification, as in

(58) Mary panicked after the accident.

(59) Mary panicked near the accident.

It seems that double-contextuality is called for here too.

Note that there are many nouns that do not allow for such doubly-contextual interpretation. For example,

(60) (*) garden in the morning

(61) $\left(^{*}\right)$ morning in the garden

are both ill-formed, each of the nouns allowing only one kind of CPP-modification. ${ }^{24}$ The nouns that do allow doubly-contextual modifications are typically nominalizations, or other nouns related to events. On a finer analysis, this should be treated by finer categorization, making explicit the above distinction, possibly using features. To keep matters simple, we proceed under the assumption that all nouns are amenable to doubly-contextualized modification.

We start by assigning the IPs, during and on in this case, another categorization, making them induce a noun modifier (in addition to the previous inducing of a sentential modifiers). Here are the details. ${ }^{25}$

』during|in|on』 $=\left.\left(N \backslash_{\diamond} N\right)\right|_{\diamond} N P$ :

$\lambda y^{e} \lambda \mathcal{N}^{(e(i(l t)))} \lambda x^{e} \lambda I^{i} \lambda M^{l} . \mathcal{N}(x)\left(F_{\text {during/in/on }}(I, \tau(y)), M\right)$

The NP a Monday is a generalized quantifier $N P \uparrow$ instantiated in this case as:

\a Monday $\llbracket=\left(N \backslash_{\diamond} N\right) \backslash_{\diamond}\left(\left(N \backslash_{\diamond} N\right) /_{\diamond} N P\right):$

$\lambda \mathcal{V}^{(e . . \vec{t})} \lambda \ldots \lambda P^{(i(l t))} \lambda I^{i} \lambda M^{l} \exists x^{e}$. Monday $(x) \wedge \tau(x) \subseteq I \wedge \sigma(x) \subseteq M \wedge \mathcal{V}(x \ldots)(P, I, M)$

When applied to on the result is:

\footnotetext{
${ }^{23}$ Indeed, in [25], TNs had the forms (in our current notation) $\lambda x^{e} \lambda I^{i} \cdot \mathbf{n}(x) \wedge \tau(x) \subseteq I$. The parallel LN would have the form $\lambda x^{e} \lambda M^{l} . \mathbf{n}(x) \wedge \sigma(x) \subseteq M$.

${ }^{24}$ We disregard the interpretation of (61) that arises in sentences like The fi rst morning in the garden, Adam said to Eve "How d'you like them apples?" as meaning "morning during their sojourn in the garden", as opposed to "mornings that occurred in the garden and not elsewhere".

${ }^{25}$ The $\diamond$ modality is required to allow cluster coordinations like every meeting [in the morning in a garden] and [in the evening at the pub], and to disallow certain overgeneralizations like *Every in the morning meeting that would otherwise arise via backward crossed composition.
} 
『on a Monday $\rrbracket=\llbracket$ a Monday $\llbracket(\rrbracket o n \rrbracket)=$

$N \backslash_{0} N: \lambda \mathcal{N}(e(i(l))) \lambda x^{e} \lambda I^{i} \lambda M^{l} \exists y^{e} . \operatorname{Monday}(y) \wedge \tau(y) \subseteq I \wedge \sigma(y) \subseteq M \wedge \mathcal{N}(x)\left(F_{o n}(I, \tau(y)), M\right)$

Recall that the noun meeting has the following category:

$$
\llbracket \text { meeting } \llbracket=N: \lambda x^{e} \lambda I^{i} \lambda M^{l} \cdot \text { meeting }(x) \wedge \tau(x) \subseteq I \wedge \sigma(x) \subseteq M
$$

Combination of meeting with on a Monday yields:

\meeting on a Monday $\rrbracket=\rrbracket$ on a Monday $\llbracket(\rrbracket$ meeting $\rrbracket)$

$$
\begin{aligned}
& =N: \lambda x^{e} \lambda I^{i} \lambda M^{l} \exists y^{e} \text {. Monday }(y) \wedge \tau(y) \subseteq I \wedge \sigma(y) \subseteq M \\
& \wedge \text { meeting }(x) \wedge \tau(x) \subseteq F_{o n}(I, \tau(y)) \wedge \sigma(x) \subseteq M
\end{aligned}
$$

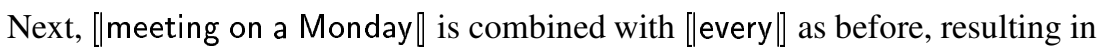

\every meeting on a Monday $\rrbracket=\llbracket$ every $\llbracket(\rrbracket$ meeting on a Monday $\rrbracket)=$

$N P^{\uparrow}: \lambda \mathcal{V}^{(e . . \vec{t})} \lambda \ldots \lambda P^{(i(l t))} \lambda I^{i} \lambda M^{l}$

$\forall x^{e} . \exists y^{e}$. Monday $(y) \wedge \tau(y) \subseteq I \wedge \sigma(y) \subseteq M \wedge$ meeting $(x) \wedge \tau(x) \subseteq F_{\text {on }}(I, \tau(y)) \wedge \sigma(x) \subseteq M$

$$
\Rightarrow \mathcal{V}(x \ldots)(P, I, M)
$$

Finally, after combining as before with \in』, in every [ ${ }_{n}$ meeting on a Monday] modifies Mary kissed John. We thus obtain

[Mary kissed John in every [meeting on a Monday] $]=$

$S: \lambda P^{(i(l t))} \lambda I^{i} \lambda M^{l} \forall x^{e} . \exists y^{e}$. Monday $(y) \wedge \tau(y) \subseteq I \wedge \sigma(y) \subseteq M \wedge$ meeting $(x) \wedge \tau(x) \subseteq F_{o n}(I, \tau(y)) \wedge \sigma(x) \subseteq M$

$\Rightarrow \exists J_{0} \exists L_{0}$.kiss (mary,john $\left.)\left(J_{0}, L_{0}\right) \wedge J_{0} \subseteq F_{\text {in }}(I, \tau(x)) \wedge L_{0} \subseteq M \wedge P\left(J_{0}, L_{0}\right)\right]$

Next, we show the effect of combining a time-wrapping preposition with a noun-modified CPP. Consider

(62) Mary kissed John [CPP before a $[n$ meeting on some morning] $]$.

As before, we have

[a meeting on some morning $\|=$

$N P^{\uparrow}: \lambda \mathcal{V}^{(e . . \vec{t})} \lambda \ldots \lambda P^{(i(l t))} \lambda I^{i} \lambda M^{l} \exists x^{e} \exists y^{e}$.morning $(y) \wedge \tau(y) \subseteq I \wedge \sigma(y) \subseteq M$

$$
\begin{aligned}
& \wedge \text { meeting }(x) \wedge \tau(x) \subseteq \bar{F}_{\text {in }}(I, \tau(y)) \wedge \sigma(x) \subseteq M \\
& \wedge \mathcal{V}(x . .)(P, I, M)
\end{aligned}
$$

In addition, we have as usual

【before $\rrbracket=(S \backslash S) /_{0} N P: \lambda x^{e} \cdot \lambda S^{\vec{t}} \lambda P^{(i(t))} \lambda I^{i} \lambda M^{l} \cdot S\left(P, F_{\text {before }}(I, \tau(x)), M\right)$

—which yields the following:

\before a meeting on some morning $\rrbracket=\rrbracket$ a meeting on some morning $\rrbracket(\rrbracket$ before $\rrbracket)$

$S \backslash S: \lambda S^{\vec{t}} \lambda P^{(i(l t))} \lambda I^{i} \lambda M^{l}$

$\exists x^{e} \exists y^{e} \cdot$ morning $(y) \wedge \tau(y) \subseteq I \wedge \sigma(y) \subseteq M \wedge$ meeting $(x) \wedge \tau(x) \subseteq F_{\text {during }}(I, \tau(y)) \wedge \sigma(x) \subseteq M$

$\wedge \mathcal{S}\left(P, F_{\text {before }}(I, \tau(x)), M\right)$

This composed modifier generates the "long reading" once combined with a modified sentence.

$\llbracket$ Mary kissed John before a meeting on some morning $\rrbracket=\rrbracket$ before a meeting on some morning $\llbracket(\rrbracket$ Mary kissed John $\rrbracket)$ $S: \lambda P^{(i(l t))} \lambda I^{i} \lambda M^{l}$

$\exists x^{e} \exists y^{e}$.morning $(y) \wedge \tau(y) \subseteq I \wedge \sigma(y) \subseteq M \wedge$ meeting $(x) \wedge \tau(x) \subseteq F_{i n}(I, \tau(y)) \wedge \sigma(x) \subseteq M$

$\wedge \exists J_{0} \exists L_{0} . \mathbf{k i s s}($ mary,john $)\left(J_{0}, L_{0}\right) \wedge J_{0} \subseteq F_{\text {before }}(I, \tau(x)) \wedge L_{0} \subseteq M \wedge P\left(J_{0}, L_{0}\right)$. 
As in the case of sentential CPP modifiers, readings which invert the scopes implied by purely applicative readings arise from nominal CPP modifier cascades like the following - cf. (15):

(63) Mary kissed John before [a meeting in some summer in every conference].

As in the case of sentential CPP modifiers, we assume that these readings involve a further category $(N \backslash N) /(N \backslash N)$ for CPPs, arising from the following category for nominal IPs. The corresponding temporal and locative categories for in/during are as follows (cf. (47) and (48):

(64) $\llbracket$ during/in $\rrbracket=\left.\left(\left.\left(N \backslash_{\diamond} N\right)\right|_{\star}\left(N \backslash_{\diamond} N\right)\right)\right|_{\star} N P: \lambda y^{e} \lambda \mathcal{A}^{((e(i(l t)))(e(i(l t))))} \lambda \mathcal{N}^{(e(i(l t)))} \lambda x^{e} \lambda I^{i} \lambda M^{l} . \mathcal{A}\left(\mathcal{N}\left(F_{d u r i n g} / i_{t}(I, \tau(y)), M\right)\right)$

(65) $\llbracket \operatorname{in} \rrbracket=\left.\left(\left.\left(N \backslash_{\diamond} N\right)\right|_{*}\left(N \backslash_{\diamond} N\right)\right)\right|_{*} N P: \lambda y^{e} \lambda \mathcal{A}^{((e(i(l t)))(e(i(l t))))} \lambda \mathcal{N}^{(e(i(l t)))} \lambda x^{e} \lambda I^{i} \lambda M^{l} . \mathcal{A}\left(\mathcal{N}(x)\left(I, F_{i n_{l}}(M, \sigma(y))\right)\right)$

\section{Sentential Complements of TPs}

We now turn to the treatment of TPPs in which the IP is complemented by a sentence. Consider the following example.

(66) Mary kissed John before Sue arrived.

For the interpretation of before (understood here as "any time before"), we again use the time-wrap function $F_{\text {before }}(I, J)$, which for $J \subseteq I$ returns the interval between the beginning of $I$ and the beginning of $J$.

The meaning of the preposition is given below.

\|before $\rrbracket=(S \backslash S) / S: \lambda S_{1}^{\vec{\tau}} \lambda S_{2}^{\vec{\tau}} \lambda P^{(i(l t))} \lambda I^{i} \lambda M^{l} \cdot S_{1}\left(\lambda J_{1}^{i} \lambda L_{1}^{l} \cdot S_{2}\left(P, F_{b e f o r e}\left(I, J_{1}\right), M\right)\right)(I, M)$

Note the "ignoring" of $L_{1}$, by which the locative extents of $S_{1}, S_{2}$ are unrelated, only their temporal extents are related.

For Sue arrived, as usual we have the following category:

$$
\text { [Sue arrived } \rrbracket=S: \lambda P^{(i(l t))} \lambda I^{i} \lambda M^{l} \exists J^{i} \exists L^{l} \text {.arrive }(\mathbf{s})(J, L) \wedge J \subseteq I \wedge L \subseteq M \wedge P(J, L)
$$

Applying the former to the latter, we get the following:

[before Sue arrived $\rrbracket=$ \before $\llbracket(\llbracket$ Sue arrived $\rrbracket)=$

$S \backslash S: \lambda S_{2}^{\vec{t}} \lambda P^{(i(l t))} \lambda I^{i} \lambda M^{l} . \exists J^{i} \exists L^{l}$.arrive $(\mathbf{s})(J, L) \wedge J \subseteq I \wedge L \subseteq M \wedge S_{2}\left(P, F_{\text {before }}(I, J), M\right)$

Finally, the TPP modifies the base sentence as before.

\Mary kissed John before Sue arrived $\rrbracket=$ [before Sue arrived $\rrbracket(\llbracket$ Mary kissed John $\rrbracket)=$ $S: \lambda P^{(i(l t))} \lambda I^{i} \lambda M^{l} \exists J_{1}^{i} \exists L_{1}^{l}$. arrive $(\mathbf{s})\left(J_{1}, L_{1}\right) \wedge J_{1} \subseteq I \wedge L_{1} \subseteq M \wedge$

$$
\exists J_{0}^{i} \exists L_{0}^{l} \cdot \mathbf{k i s s}(\mathbf{m a r y}, \mathbf{j o h n})\left(J_{0}, L_{0}\right) \wedge J_{0} \subseteq F_{\text {before }}\left(I, J_{1}\right) \wedge L_{0} \subseteq M \wedge P\left(J_{0}, L_{0}\right)
$$

This logical form can be paraphrased as Within the contextual extents, there exists an event-time and an eventlocation of an arrival (by Sue), such that before that event-time there exists another event-time, and event-location, of a kissing (of John by Mary). Note how the $P$-argument of the meaning of Sue arrived "captures" the modified sentence and restricts its event-extents. This "implements" the function of a sentence as a preposition-complement.

We note that this interpretation is one place where our simplification in using existential quantification over the event extents $J_{1}, L_{1}$ etc. comes back to haunt us. ${ }^{26}$ Since the models for Mary kissed John before Sue arrived for

\footnotetext{
${ }^{26}$ The present theory does not explain the fact that example (66) forces a meaning involving a unique arrival of Sue within the contextual interval. We assume that this constraint arises from the aspectual character of Sue arrived as an achievement whose consequent state of Sue's being present precludes any further instances of Sue arriving - that is, from the same aspectual character that also notoriously rules out examples like * Sue arrived all night. We leave the integration of a theory of such aktionsarten with the present theory as a problem for further research.
} 
the last time are identical to those for the above interpretation, the theory technically makes the false implication that this sentence means the same as Mary kissed John before Sue arrived. We have already noted that the existentials are merely a placeholder for definite quantification of the kind given for the article the in figures 3 and 4. Such definite formulæ avoid this problem, which we will continue to ignore for present purposes.

For the attachment of in the morning as a (second) CPP-modifier of Mary kissed John, our previously described cascading method yields the "short reading" for the following example:

(67) Mary kissed John before Sue arrived in the morning.

The "long reading"is obtained by an attachment of in the morning to Sue arrived, having the modified sentence Sue arrived in the morning as the complement of before. This reading is analogous to the effect of noun-modification by CPPs discussed in Section 4.

The application of a type-raised NP \the morning \ to the preposition meaning yields the following category for the adjunct:

\in the morning $\llbracket=S \backslash S: \lambda S_{2}^{\vec{t}} \cdot \lambda P^{(i(l t))} \lambda I^{i} \lambda M^{l} \exists ! x^{e} \cdot \operatorname{morning}(x) \wedge \tau(x) \subseteq I \wedge \sigma(x) \subseteq M \wedge S_{2}\left(P, F_{i n_{t}}(I, \tau(x)), M\right)$

This can combine with a sentence such as the following:

[Sue arrived》] $=S: \lambda P^{(i(l t))} \lambda I^{i} \lambda M^{l} \exists J^{i} \exists L^{l}$.arrive(s) $(J, L) \wedge J \subseteq I \wedge L \subseteq M \wedge P(J, L)$

The result is as follows:

\Sue arrived in the morning $\rrbracket=\llbracket$ in the morning $\llbracket(\rrbracket$ Sue arrived $\rrbracket)=S$ :

$$
\begin{aligned}
\lambda P^{(i(l t))} \lambda I^{i} \lambda M^{l} \exists ! x^{e} \text {.morning }(x) \wedge \tau(x) \subseteq I \wedge \sigma(x) \subseteq M \wedge \\
\exists J^{i} \exists L^{l} \text {.arrive }(\mathbf{s})(J, L) \wedge J \subseteq F_{i n_{t}}(I, \tau(x)) \wedge L \subseteq M \wedge P(J, L)
\end{aligned}
$$

This sentence can in turn become the complement of a preposition like before:

\before Sue arrived in the morning $\rrbracket=\llbracket$ before $\rrbracket(\rrbracket$ Sue arrived in the morning $\rrbracket)$

$=S \backslash S: \lambda S^{\vec{t}} \lambda P^{(i(l))} \lambda I^{i} \lambda M^{l} \exists ! x^{e}$.morning $(x) \wedge \tau(x) \subseteq I \wedge \sigma(x) \subseteq M \wedge$

$\exists J^{i} \exists L^{l}$.arrive $(\mathbf{s})(J, L) \wedge J \subseteq F_{\text {int }_{t}}(I, \tau(x)) \wedge L \subseteq M \wedge \mathcal{S}\left(P, F_{\text {before }}(I, J), M\right)$

Clearly, when combined with a modified sentence this generates the "long reading". 27

\subsubsection{Veridicality of before}

The observant reader will have noticed, at this stage, a certain symmetry between the meanings of after and before. For both, the meanings are veridical wherby both $\phi$ be fore $\psi$ and $\psi$ after $\phi$ imply both $\phi$ and $\psi$ (at the appropriate contextual arguments). It has been argued, based on examples like

(68) Mozart died before he finished the Requiem

(69) The police defused the bomb before it exploded

("preemptive-causative") that before has also a non-veridical meaning, manifested in that Mozart finished the Requiem is not implied by (68), nor is the bomb exploded implied by (69). The strongest argument for this non-veridicality seems to come from liscencing polarity items (see [12, 13]).

We note that the architecture of our account is not incompatible with the incorporation of a second lexical meaning of before, with a modal force in the form of a second $F_{\text {before }}$ (or the derivation ${ }^{28}$ of both from a common core

\footnotetext{
${ }^{27}$ If the definite in (67) is replaced by an indefinite such as on some weekday, then the problem alluded to in connection with (66) of spurious equivalence with before Sue arrived on some morning for the last time would recur, since the existential would outscope the spatio-temporal definite existentials introduced by arrive. However, before seems to demand definites.

${ }^{28} \mathrm{~A}$ recent attempt for such a derivation appears in [4], with a detailed discussion of veridicality of those prepositions. However, the discussion there relates to states, not to events, and there is strict adherence to type-driven derivations only.
} 
meaning). This would mean using a branching-time temporal ontology, i.e., some Kripke structure, in contrast to the linear-time used here. However, as we adhere to a strict type-driven approach, such an extension will be best handled once a good characterization is found for the verbs in the main clause that give rise to such worldknowledge dependent readings ("died-like") verbs, or to the relation between this verb and the verb in the temporal clause complementing the preposition before ("diffuse-explode" pairs). To date, we are not aware of any such characterizations. Type-theoretically, the above examples cannot be told apart from veridical readings in

(70) Verdi smiled before he finished the Requiem

(71) The dog smelled the bomb before it exploded

It is not at all clear how such a characterization might look like. Can semantics really reflect (whose?) beliefs about the possibility of resurrection?

\subsection{Quantifi ed sentences as temporal preposition complements}

Let us consider next the effect of a (universal) quantifier in the sentence complementing a TP - for example, a universally quantified subject:

(72) After every girl smiled, Mary applauded.

For the (preposed) preposition we have:

\after $\rrbracket=(S / S) / S: \lambda S_{1}^{\vec{t}} \lambda S_{2}^{\vec{t}} \lambda P^{(i(l t))} \lambda I^{i} \lambda M^{l} . S_{1}\left(\lambda J_{1}^{i} \lambda L_{1}^{l} \cdot S_{2}\left(P, F_{a f t e r}\left(I, J_{1}\right), M\right), I, M\right)$

We repeat here the sentential complement (28), without the redundant contextual inclusions in \girl】.

[Every girl smiled $\|=\llbracket$ every $\operatorname{girl} \rrbracket(\rrbracket$ smiled $\rrbracket)=$

$$
S: \lambda P^{(i(l t))} \lambda I^{i} \lambda M^{l} \forall x^{e} \cdot \operatorname{girl}(x) \Rightarrow \exists J^{i} \exists L^{l} . \operatorname{smile}(x)(J, L) \wedge J \subseteq I \wedge L \subseteq M \wedge P(J, L)
$$

Combining, we get

\After every girl smiled $\rrbracket=\llbracket$ after $\llbracket(\rrbracket$ every girl smiled $\rrbracket)=$ $S / S: \lambda S_{2}^{\vec{t}} \lambda P^{(i(l t))} \lambda I^{i} \lambda M^{l} \forall x$.girl $(x) \Rightarrow \exists J^{i} \exists L^{l}$.smile $(x)(J, L) \wedge J \subseteq I \wedge L \subseteq M \wedge S_{2}\left(P, F_{\text {after }}(I, J), M\right)$

This yields the "multiple applauses" reading, where there is a separate applause (with its own event-time and event-location) per girl smiling.

In order to get the "single applause" reading, where there is one applause after all girls have smiled, we need another lexical meaning for after. It will create an event-time for a "cumulative" event, including the event-times of all individual smiling events. The event-time of the "applause event" will be located after this fictitious cumulative event. For this, we semi-finalize the sentential complement of after. We have

Пafter $\rrbracket=(S / S) / S$ :

$\lambda S_{1}^{\vec{t}} \lambda S_{2}^{\vec{t}} \lambda P^{(i(l t))} \lambda I^{i} \lambda M^{l} \exists J_{2}^{i} \exists L_{2}^{l} . J_{2} \subseteq I_{2} \wedge L_{2} \subseteq M_{2} \wedge S_{1}\left(\lambda I \lambda J\right.$.true $\left., J_{2}, L_{2}\right) \wedge S_{2}\left(P, F_{\text {after }}\left(I, J_{2}\right), M\right)$.

By combining with [every girl smiled|], we get

\after every girl smiled $\rrbracket=$ |after $\llbracket($ (every girl smiled $\rrbracket)=$ $S / S: \lambda S_{2}^{\vec{t}} \lambda P^{(i(l t))} \lambda I^{i} \lambda M^{l} \exists J_{2}^{i} \exists L_{2}^{i} . J_{2} \subseteq I \wedge L_{2} \subseteq M$

$\wedge\left[\forall x\right.$.girl $(x) \Rightarrow \exists J_{1} \exists L_{1}$.smile $\left.(x)\left(J_{1}, L_{1}\right) \wedge J_{1} \subseteq J_{2} \wedge L_{1} \subseteq M\right]$

$\wedge \mathcal{S}_{2}\left(P, F_{\text {after }}\left(I, J_{2}\right), M\right)$

Note how $F_{\text {after }}$ locates the event denoted by $S_{2}$ after the "cumulative" $J_{2}$ (which contains all the $J_{1}$ s), embodying the 'single event' reading. Some justification for the assumption of a separate meaning for a preposition yielding a "single event" reading is provided by the observation that some other prepositions, such as once, seem to have only this reading, as in

(73) Once every girl (had) smiled, Mary applauded. 


\section{Coordination of contextual preposition-phrases}

In this section we treat the various form of coordination involving CPPs, both constituent and non-constituent coordination. Both have long been considered as strongholds of categorial grammar in its various forms. As we see, this issue brings to light one major point, the question of how many events are involved in a (binary) CPPcoordination. It also highlights coordinability of temporal and locative CPPs. Note that here we again capitalize on the CCG power; the direct approach in [25], without type-raising, would not yield the required results.

The use of multimodal CCG allows us to treat coordination as arising from a proclitic category for conjunctions $\left(X \backslash_{\star} X\right) /_{\star} X$. For example:

$$
\text { [and } \|=\left.\left(X \backslash_{\star} X\right)\right|_{\star} X: \lambda x_{1} \lambda x_{2} \cdot x_{1} \wedge x_{2}
$$

Here $x_{1}, x_{2}$ range over arbitrary conjoinable terms of the same semantic type. If the type is $t$, then ' $\wedge$ ' is standard conjunction. For higher-order types, we have the usual (pointwise) recursive definition as in [22]

$$
\lambda x . X \wedge \lambda y . Y={ }^{d f} \cdot \lambda z . X[z / x] \wedge Y[z / y]
$$

where $X[z / x]$ denotes substituting $z$ for all free occurrences of $x$ in $X$.

\subsection{Constituent Coordination of contextual preposition-phrases}

Consider the following sentences:

(74) Mary kissed John during some meeting and before some lunch-break.

(75) Mary kissed John on some morning and in some garden.

For (75), we get the following meaning for [on some morning and in some garden] via the above conjunction category with $X=S \backslash S$ :

【on some morning $\llbracket \wedge \llbracket$ in some garden $\rrbracket=$

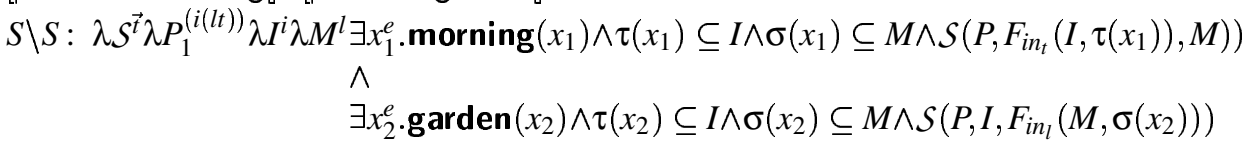

And by applying to 『Mary kissed John』, we get

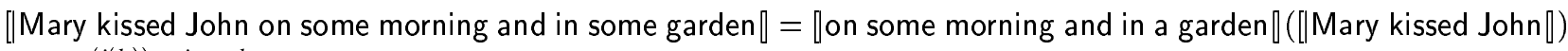
$S: \lambda P^{(i(l t))} \lambda I^{i} \lambda M^{l}\left[\exists x_{1}^{e}\right.$.morning $\left(x_{1}\right) \wedge \tau\left(x_{1}\right) \subseteq I \wedge \sigma\left(x_{1}\right) \subseteq M \wedge$

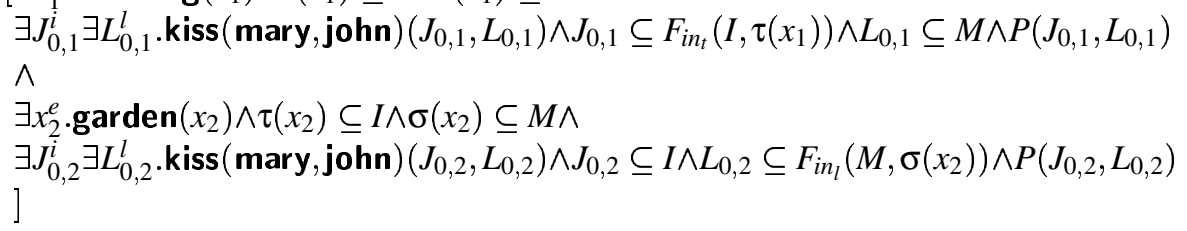

Similarly, for (74), we get

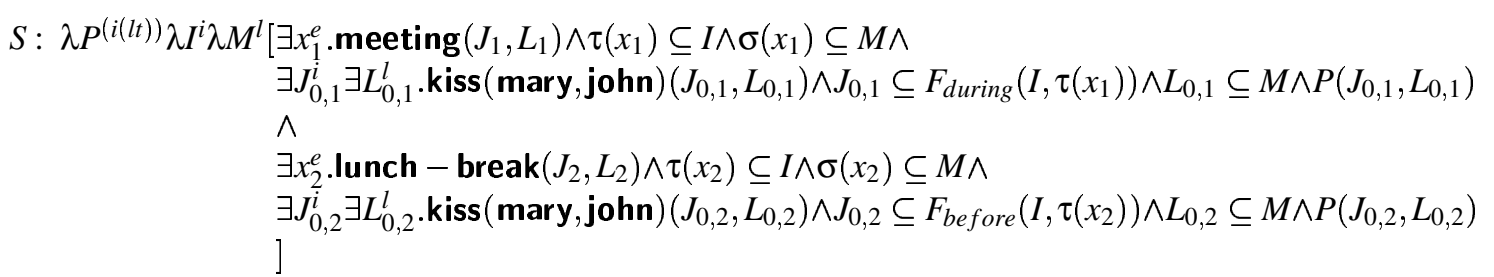


These meanings leave to pragmatics the question mentioned earlier of how many kissing events are implicated by a coordinated-CPP modification like (75) or (74). The semantics provides a double existential quantification on event-times and even-locations, but the pairs $\left(J_{0,1}, L_{0,1}\right)$ and $\left(J_{0,2}, L_{0,2}\right)$ satisfy compatible (sometimes, even identical) constraints, contributed by the two, possibly different IPs.

If, as seems often to be the case, it is possible to consistently identify the existentially-quantified event-extents, the natural reading is often to do so-that is, to view the sentences as describing one event, having two non-conflicting modifications. However, this appears to be simply a pragmatic bias: whenever world knowledge would render such identification inconsistent, a double-event reading is available For example, knowing that mornings and evenings are disjoint in their temporal extents, and that Jerusalem and Edinburgh are disjoint in their spatial extents, is enough to make the double-event reading emerge in the following sentences, yielding two kissing-events.

(76) Mary kissed John in the morning and in the evening.

(77) Mary kissed John in Jerusalem and in Edinburgh.

We therefore conclude that our theory is correct to allow both readings.

For $N P$-coordination within TPPs, we again encounter the one-event/double-event phenomenon, but this time without the contribution of different restrictions due to the preposition itself, which is one and the same for the two modifications. Consider the following example.

(78) Mary kissed John in [[every meeting] and [some departure]].

We have

\every meeting $\llbracket \wedge \llbracket$ some departure $\rrbracket=S /(S \backslash N P)$ :

$\lambda \mathcal{V}^{(e t)} \lambda P^{(i(l t))} \lambda I^{i} \lambda M^{l} \forall x_{1}^{e}$.meeting $\left(x_{1}\right) \wedge \tau\left(x_{1}\right) \subseteq I \wedge \sigma\left(x_{1}\right) \subseteq M \Rightarrow \mathcal{V}\left(x_{1}\right)(P, I, M)$

$\wedge$

$\exists x_{2}^{e}$.departure $\left(x_{2}\right) \wedge \tau\left(x_{2}\right) \subseteq I \wedge \sigma\left(x_{2}\right) \subseteq M \wedge \mathcal{V}\left(x_{2}\right)(P, I, M)$

Note that those two $N P$-meanings are assumed to be similarly raised within a coordination. By combining with the preposition meaning \in|], and then with the modificant sentence \Mary kissed John】, we get for (78)

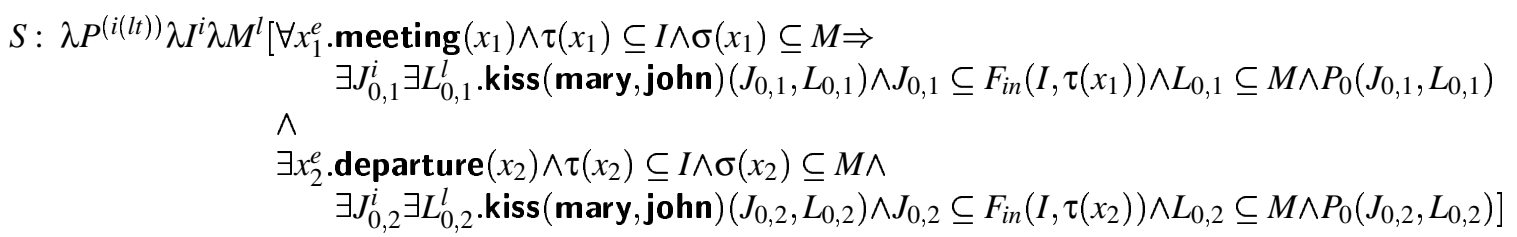

Similarly, we consider the coordination of the prepositions themselves. Consider the sentence

(79) Mary kissed John before and after every meeting.

Recalling the meanings of the (time-wrapping) prepositions, we have

$\llbracket$ after $\rrbracket=(S \backslash S) / N P:=\lambda x^{e} . \lambda \mathcal{S}^{\vec{t}} \lambda P^{(i(l t))} \lambda I^{i} \lambda M^{l} . S\left(P, F_{\text {after }}(I, \tau(x)), M\right)$

Øbefore $\rrbracket=(S \backslash S) / N P:=\lambda x^{e} \lambda \overrightarrow{S^{t}} \lambda P^{(i(l t))} \lambda I^{i} \lambda M^{l} . S\left(P, F_{\text {before }}(I, \tau(x)), M\right)$

Coordinating, we get

ఐbefore and after $\rrbracket=(S \backslash S) / N P$ :

$\lambda x^{e} \lambda \mathcal{S}^{\vec{\imath}} \lambda P^{(i(l t))} \lambda I^{i} \lambda M^{l} . S\left(P, F_{\text {before }}(I, \tau(x)), M\right)$

$\wedge$

$\mathcal{S}\left(P, F_{\text {after }}(I, \tau(x)), M\right)$ 
Applying (the appropriately raised) \every meeting』 to the above, we get the following:

[before and after every meeting $\rrbracket=$ =every meeting $\rrbracket(($ before and after $\rrbracket)=S \backslash S$ :

$$
\begin{aligned}
& \left.\lambda \mathcal{S}^{t}\right) \lambda P^{(i(l t))} \lambda I^{i} \lambda M^{l} . \forall x \text {.meeting }(x) \wedge \tau(x) \subseteq I \wedge \sigma(x) \subseteq M \Rightarrow \\
& {\left[\mathcal{S}\left(P, F_{\text {before }}(I, \tau(x)), M\right)\right)} \\
& \wedge \\
& \left.\mathcal{S}\left(P, F_{\text {after }}(I, \tau(x)), M\right)\right)
\end{aligned}
$$

Finally, modifying \Mary kissed John】, we get

\Mary kissed John before and after every meeting $\rrbracket=$

$S: \lambda P^{(i(l t))} \lambda I^{i} \lambda M^{l} \forall x^{e}$.meeting $(x) \wedge \tau(x) \subseteq I \wedge \sigma(x) \subseteq M \Rightarrow$

$$
\begin{aligned}
& {\left[\exists J_{0,1}^{i} \exists L_{0,1}^{l} \cdot \text {. kiss (mary,john }\right)\left(J_{0,1}, L_{0,1}\right) \wedge J_{0,1} \subseteq F_{\text {before }}(I, \tau(x)) \wedge L_{0,1} \subseteq M \wedge P\left(J_{0,1}, L_{0,1}\right)} \\
& \left.\left.\hat{\exists} J_{0,2}^{i} \exists L_{0,2}^{l} \text {.kiss(mary,john }\right)\left(J_{0,2}, L_{0,2}\right) \wedge J_{0,2} \subseteq F_{\text {after }}(I, \tau(x)) \wedge L_{0,2} \subseteq M \wedge P\left(J_{0,2}, L_{0,2}\right)\right]
\end{aligned}
$$

\subsection{Non-constituent coordination within CPPs}

The following "non-constituent" coordination is handled as smoothly in categorial grammar as "constituent coordination":

(80) Mary kissed John [before every] and [after some] meeting.

As before, we have

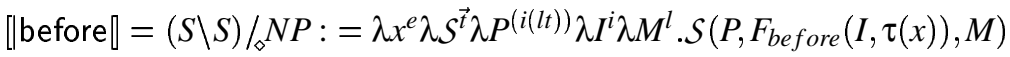

For \every】, we take its category as typed-raised over that of a preposition.

$\llbracket$ every $\rrbracket=\left((S \backslash S) \backslash_{0}\left((S \backslash S) /_{0} N P\right)\right) /_{0} N$ :

$$
\lambda \mathcal{N}^{(e(i(l t)))} \lambda \mathcal{F}^{(e(\vec{t} t))} \lambda \mathcal{S}^{i} \lambda P^{(i(l t))} \lambda I^{i} \lambda M^{l} \forall x^{e} \cdot \mathcal{N}(x)(I, M) \Rightarrow \mathcal{F}(x)(S, P, I, M)
$$

By raising the preposition over (the already raised) determiner ${ }^{29}$, we get composable categories; composing the meanings, we get

\before every $\llbracket=(S \backslash S) /_{0} N$ :

$\lambda \mathcal{N}^{(e(i(l t)))} \lambda \mathcal{S}^{\vec{t}} \lambda P^{(i(l t))} \lambda I^{i} \lambda M^{l} \forall x^{e} . \mathcal{N}(x)(I, M) \Rightarrow \mathcal{S}\left(P, F_{\text {before }}(I, \tau(x)), M\right)$

Similarly,

\after some $\rrbracket=(S \backslash S) /_{0} N$ :

$\lambda \mathcal{N}(e(i(l))) \lambda \mathcal{S}^{\vec{t}} \lambda P^{(i(l t))} \lambda I^{i} \lambda M^{l} \exists x^{e} \cdot \mathcal{N}(x)(I, M) \wedge \mathcal{S}\left(P, F_{a f t e r}(I, \tau(x)), M\right)$

Coordinating, we get

\before every and after some $\llbracket=(S \backslash S) /_{0} N$ :

$\lambda \mathcal{N}^{(e(i(l t)))} \lambda \mathcal{S}^{\vec{t}} \lambda P^{(i(l t))} \lambda I^{i} \lambda M^{l} \forall x_{1}^{e} \cdot \mathcal{N}\left(x_{1}\right)(I, M) \Rightarrow \mathcal{S}\left(P, F_{\text {before }}\left(I, \tau\left(x_{1}\right)\right), M\right)$

$\wedge$

$$
\exists x_{2}^{e} \cdot \mathcal{N}\left(x_{2}\right)(I, M) \wedge \mathcal{S}\left(P, F_{\text {after }}\left(I, \tau\left(x_{2}\right)\right), M\right)
$$

\footnotetext{
${ }^{29}$ Note that this is a departure from standard CCG practice, where only argument categories are raised. Some restriction is still needed here, to prevent overgeneration.
} 
Applying to $N$ : \meeting $\rrbracket$, we get

[before every and after some meeting $\llbracket=(S \backslash S)$ :

$\lambda \mathcal{S}^{\vec{t}} \lambda P^{(i(l))} \lambda I^{i} \lambda M^{l} \forall x_{1}^{e}$.meeting $\left(x_{1}\right) \wedge \tau\left(x_{1}\right) \subseteq I \wedge \sigma\left(x_{1}\right) \subseteq M \Rightarrow S\left(P, F_{\text {before }}\left(I, \tau\left(x_{1}\right)\right), M\right)$

$\wedge$

$\exists x_{2}^{e}$.meeting $\left(x_{2}\right) \wedge \tau\left(x_{2}\right) \subseteq I \wedge \sigma\left(x_{2}\right) \subseteq M \wedge \mathcal{S}\left(P, F_{a f t e r}\left(I, \tau\left(x_{2}\right)\right), M\right)$

Finally, modifying $S$ : [Mary kissed John』, we get

[Mary kissed John before every and after some meeting $]=S$ :

$\lambda P^{(i(l t))} \lambda I^{i} \lambda M^{l} \forall x_{1}^{e}$.meeting $\left(x_{1}\right) \wedge \tau\left(x_{1}\right) \subseteq I \wedge \sigma\left(x_{1}\right) \subseteq M \Rightarrow$

$\exists J_{0,1}^{i} \exists L_{0,1}^{l} \cdot$ kiss (mary,john $\left.)\left(J_{0,1}, L_{0,1}\right) \wedge J_{0,1} \subseteq F_{\text {before }}\left(I, \tau\left(x_{1}\right)\right) \wedge L_{0,1} \subseteq M\right) \wedge P\left(J_{0,1}, L_{0,1}\right)$

$\wedge$

$\exists x_{2}^{e} \cdot$ meeting $\left(x_{2}\right) \wedge \tau\left(x_{2}\right) \subseteq I \wedge \sigma\left(x_{2}\right) \subseteq M \wedge$

$\exists J_{0,2}^{i} \exists L_{0,2}^{l} \cdot$ kiss (mary,john $\left.)\left(J_{0,2}, L_{0,2}\right) \wedge J_{0,2} \subseteq F_{\text {after }}\left(I, \tau\left(x_{2}\right)\right) \wedge L_{0,2} \subseteq M\right) \wedge P\left(J_{0,2}, L_{0,2}\right)$

It is also possible to coordinate TPPs with LPPs, as shown by the following examples. We skip the formal details, which by now should be clear.

(81) Mary panicked [after and near] the accident.

(82) The suspect left the train a kilometer away from, and a few minutes before, the explosion that destroyed it.

\subsection{Further restrictions on scope under coordination}

As in the CCG accounts of quantifier coordination in [21] and [30], our account predicts both a single-applause/singlecheering reading, and a multiple-applause/multiple-cheering reading, but excludes mixed readings like singleapplause/multiple cheering, for the following example:

(83) After every girl smiled, Mary applauded and Sue cheered.

A similar predition follows for

(84) After every girl smiled and every boy giggled, Mary applauded.

Furthermore, for longer-cascade coordinations, since out-of-scope modification is calculated by compositions within each conjunct separately, we get all the expected readings for

(85) Mary kissed John during every meeting in some summer and in every barn-dance in a winter.

These examples by no means exhaust the variety of ways in which CCG correctly projects logical forms under coordination of both constituent and non-constituent fragments.

\section{Conclusions}

In this paper, we have presented a unified semantic theory of contextual (temporal and locative) preposition phrases. The theory is cast within the CCG categorial grammar framework, enforcing its strict type-driven syntax-semantics interface. The basic ingredients of the temporal semantics of TPPs presented in [25] have been adopted, and made fit for incorporation into a CCG grammar. We have taken advantage of the repertoire of Combinatory rules provided by $\mathrm{CCG}$, in particular the various functional composition operations and type-raising, to simplify the structure of the semantics, avoiding unorthodox semantic operations, excessive lexicalized ambiguity (e.g., of verbs and 
sentences), and highlighting the relationship between adjuncts modifying heads (in contrast to combining heads with complements). In particular, by an appeal to crossed-composition, we have been able to restrict the available out-of-order interpretations of cascaded CPP modifications. In addition, we have been able to account smoothly for (constituent and nonconstituent) coordination within CPPs, highlighting the issue of one event vs. two events in their interpretation, and transcending the methods employed in [25].

Under some simplification (such as allowing every noun to be modifiable both temporally and spatially), we achieved a striking uniformity between the two contextual extents, characterizing events. This view is reinforced by the coordinatability of CPPs modifying the different extents. We have as yet no explanation for the fact that temporal prepositions tend to be more compatible with sentential complements than are locative ones.

As already indicated in [24], the theory extends naturally to stative sentence modification by temporal CPPs. We defer a full categorial grammar account of stative sentences to another occasion. Another extension of the theory, pertaining to cumulative readings (using plural times/locations), as in

(86) Andrew Johnson and Lyndon B. Johnson took office after Lincoln and Kennedy died.

(87) The gangster buried the witnesses in basements and grage dumps

is presented in [1]. In (86), there ia reading of "respectvely"; in (87), some witnesses were buried in basements, and some in garbage dumps, for the sentence to be true. A "cumulative conjunction" is employed for the latter. A natural question arising from our treatment of CPP-modification (raised also by a referee) is, how is this treatment related to other PP-modifications? And if the treatments differ, is this difference justified? One natural class of PPs suitable for such a comparison are instrumental PPs, or source/goal PPs, as in

(88) John opened a door with every key

(89) John drove from every airport to some beach.

The main difference between such PPs and CPPs is, that in contrast to the latter, such PPs do not cascade. Thus, the elaborate mechanism of contextual variables, mediating contextual information to facilitate correct meaning assignments to such cascades, is not needed for those PPS. In particular, the noun within such a PP can preserve its traditional (et) type. In [9], a treatment of such PPs is proposed, where the contribution of an instrumental PP, for example, is viewed as an arity expander", by which the meaning of the head-verb has a slot for an instrument argument (or for any other PP-suplied argument). In the lexical meaning of the verb, all those arguments are existentially quantified, leaving the verb as meaning as a relation between its complements. The semantic contribution of a PP is, by means of applying a novel unbinding logical operator, to unbind the initial existential quantifier, and rebind by the quantifier in the meaning of the PP-determiner. Thus, the meaning of John opened a door, namely

$$
\exists x^{e} \exists z^{e} \text {.door }(x) \wedge \text { open }(\text { John }, x, z)
$$

is converted by the PP with every key, via unbinding-rebinding $z$, to

$$
\forall z^{e} \cdot \operatorname{key}(z) \Rightarrow i \exists x^{e} \cdot \operatorname{door}(x) \wedge \operatorname{open}(\operatorname{John}, x, z)
$$

Hence, the approaches to other PPs and CPPs are not unrelated.

\section{Appendix}

This appendix presents the whole lexicon considered in this paper.

Intransitive verbs $v: S \backslash N P: \lambda x^{e} \lambda P^{(i(t))} \lambda I^{i} \lambda M^{l} \exists J^{i} \exists L^{l} \cdot \mathbf{v}(x)(J, L) \wedge J \subseteq I \wedge L \subseteq M \wedge P(J, L)$.

Transitive verbs $v:(S \backslash N P) / N P: \lambda y^{e} \lambda x^{e} \lambda P^{(i(l t))} \lambda I^{i} \lambda M^{l} \exists J^{i} \exists L^{l} \cdot \mathbf{v}(y, x)(J, L) \wedge J \subseteq I \wedge L \subseteq M \wedge P(J, L)$.

Prepositions: - NP-complemented prepositions, modifying sentences.

Common categories: $(S \backslash S) /_{0} N P,\left(S / /_{*} S\right) / 0 N P$. 
$\llbracket$ during/in/after $\rrbracket=\lambda x^{e} \lambda \overrightarrow{S^{t}} \lambda P^{(i(l t))} \lambda I^{i} \lambda M^{l} . S\left(P, F_{\text {during/int } / \text { after }}(I, \tau(x)), M\right.$

\in/behind $\llbracket=\lambda x^{e} . \lambda \overrightarrow{S^{t}} \lambda P^{(i(l t))} \lambda I^{i} \lambda M^{l} \mathcal{S}\left(P, I, F_{i n_{l} / \text { behind }}(M, \sigma(x))\right)$

Common categories: $\left((S \backslash S) /_{*}(S \backslash S)\right) /_{*} N P,\left((S / S) /_{*}(S / S)\right) /_{*} N P$.

$\llbracket$ during/in $\rrbracket=\lambda x^{e} \lambda \mathcal{A}^{(\overrightarrow{t t})} \lambda \overrightarrow{S^{t}} \lambda P^{(i(l t))} \lambda I^{i} \lambda M^{l} . \mathcal{A}\left(\mathcal{S}\left(P, F_{\text {during } / \text { int }}(I, \tau(x)), M\right)\right)$

\in $/$ behind $\llbracket=\lambda x^{e} \lambda \mathcal{A}^{(\overrightarrow{i t})} \lambda \overrightarrow{S^{t}} \lambda P^{(i(l t))} \lambda I^{i} \lambda M^{l} . \mathcal{A}\left(S\left(P, I, F_{\text {in }_{\text {behind }}},(M, \sigma(x))\right)\right)$

- NP-complemented preposition, modifying nouns.

Common category: $\left(N \backslash_{0} N\right) /_{0} N P$.

$\llbracket$ during $\mid$ on $\rrbracket=\lambda y^{e} \lambda \mathcal{N}(e(i(l t))) \lambda x^{e} \lambda I^{i} \lambda M^{l} . \mathcal{N}(x)\left(F_{\text {during } / o n}(I, \tau(y)), M\right)$

Common category: $\left(\left(N \backslash_{0} N\right) /_{*}\left(N \backslash_{0} N\right)\right) /_{*} N P$

$\llbracket \operatorname{in} \rrbracket=\lambda y^{e} \lambda \mathcal{A}^{((e i(i(t)))(e(i(l t))))} \lambda \mathcal{N}^{(e(i(l t)))} \lambda x^{e} \lambda I^{i} \lambda M^{l} . \mathcal{A}\left(\mathcal{N}(x)\left(I, F_{i n_{l}}(M, \sigma(y))\right)\right)$

- sentence-complemented preposition, modifying sentences.

Common categories: $(S \backslash S) / /_{0} S,(S / S) /_{0} S$.

- For "multiple events" reading: 『before $=\lambda S_{1}^{\vec{t}} \lambda S_{2}^{\vec{t}} \lambda P^{(i(l t))} \lambda I^{i} \lambda M^{l} . S_{1}\left(\lambda J_{1} \lambda L_{1} . S_{2}\left(P, F_{\text {before }}\left(I, J_{1}\right), M\right)\right)(I, M)$

- For "single event" readings: $\llbracket$ after $\rrbracket=\lambda \mathcal{S}_{1}^{\vec{\imath}} \lambda \mathcal{S}_{2}^{\vec{t}} \lambda P^{(i(l t))} \lambda I^{i} \lambda M^{l} \exists J_{2}^{i} \exists L_{2}^{l} . J_{2} \subseteq I_{2} \wedge L_{2} \subseteq M_{2} \wedge \mathcal{S}_{1}\left(\lambda I \lambda J\right.$.true, $\left.J_{2}, M\right) \wedge \mathcal{S}_{2}\left(P, F_{\text {after }}\left(I, J_{2}\right), M\right)$.

Determiners: Common category: $N P \uparrow /{ }_{0} N$.

$$
\begin{aligned}
& \text { \every } \square=\lambda \mathcal{N} \mathcal{N}^{(e(i(l t)))} \lambda \mathcal{V}^{(e . . \vec{t})} \lambda \ldots \lambda P^{(i(l t))} \lambda I^{i} \lambda M^{l} . \forall x^{e} . \mathcal{N}(x)(I, M) \Rightarrow \mathcal{V}(x \ldots)(P, I, M) \\
& \llbracket \mathrm{a} / \mathrm{some} \rrbracket=\lambda \mathcal{N}^{(e(i(l t)))} \lambda \mathcal{V}^{(e . . \vec{t})} \lambda \ldots \lambda P^{(i(l t))} \lambda I^{i} \lambda M^{l} . \exists x^{e} . \mathcal{N}(x)(I, M) \wedge \mathcal{V}(x \ldots)(P, I, M) \\
& \llbracket \text { the } \rrbracket=\lambda \mathcal{N} \mathcal{N}^{(e(i(l t)))} \lambda \mathcal{V}^{(e . . \vec{t})} \lambda \ldots \lambda P^{(i(l t))} \lambda I^{i} \lambda M^{l} . \exists ! x^{e} . \mathcal{N}(x)(I, M) \wedge \exists x^{e} \cdot \mathcal{N}(x)(I, M) \wedge \mathcal{V}(x \ldots)(P, I, M) \\
& \llbracket \mathrm{no} \rrbracket=\lambda \mathcal{N}^{(e(i(t)))} \lambda \mathcal{V}^{(e . . \vec{t})} \lambda \ldots \lambda P^{(i(l t))} \lambda I^{i} \lambda M^{l} . \forall x^{e} . \mathcal{N}(x)(I, M) \Rightarrow \neg \mathcal{V}(x \ldots)(P, I, M)
\end{aligned}
$$

Nouns: Common category: $N$.

$$
\llbracket \mathrm{n} \rrbracket=\lambda x^{e} \lambda I^{i} \lambda M^{l} \cdot \mathbf{n}(x) \wedge \tau(x) \subseteq I \wedge \sigma(x) \subseteq M
$$

Proper names: Common category: $N P \uparrow$.

$$
\llbracket \text { Mary } \rrbracket=\lambda \mathcal{V}^{(e \vec{t})} \lambda P^{(i(t))} \lambda I^{i} \lambda M^{l} . \mathcal{V}(\text { mary })(P, I, M) .
$$

Relative pronouns: Common category: $\left(N \backslash_{0} N\right) /_{0}(S \backslash N P),\left(N \backslash_{0} N\right) /_{0}(S / N P)$.

$$
\llbracket \text { that } \rrbracket=\lambda \mathcal{P}^{(e \vec{t})} \lambda \mathcal{N} \mathcal{N}^{(e(i(l t)))} \lambda x^{e} \lambda P^{(i(l t))} \lambda I^{i} \lambda M^{l} \exists J^{i} \exists L^{l} . \mathcal{N}(x)(I, M) \wedge \mathbf{v}(x)(J, L) \wedge J \subseteq I \wedge L \subseteq M \wedge P(J, L) .
$$

Conjunctions: Common category: $\left(X \backslash_{*} X\right) /_{*} X$.

$$
\text { \and } \square=\left.\left(X \backslash_{*} X\right)\right|_{*} X: \lambda x_{1} \lambda x_{2} \cdot x_{1} \wedge x_{2}
$$

\section{Acknowledgments}

The research reported in this paper was carried out at Edinburgh University, Division of Informatics, funded by the Engineering and physical sciences research council (EPSRC), grant number GR/R82838/01. At the Technion, the work of the first author was partially funded by the grant for the promotion of research, and by a grant by FIRST, the fund for interdisciplinary research administered by the Israeli Accademy for Sciences and Art. We thank Ron Artstein, Jason Baldridge, Dick Oehrle, and Ian Pratt-Hartmann for helpful discussions, and David Brée for some information on preposition phrases in a corpus. We also thank two anonymous referees for pointing out several issues, the explicit treatment of which improved, we hope, the presentation. 


\section{References}

[1] Ron Artstein and Nissim Francez. Plural time and temporal modification. In Paul Dekker and Robert van Rooy, editors, 14th Amsterdam Colloquium, Amsterdam, The Netherlands, 2003. Amsterdam University.

[2] Jason Baldridge. Lexically specified derivational control in Combinatory Categorial Grammar. PhD thesis, University of Edinburgh, 2002.

[3] Jason Baldridge and Geert-Jan Kruijff. Multi-modal combinatory categorial grammar. In Proceedings of the 10th aanual meeting of the Eoropean Association for Computational Linguistics (EACL), Budapest, April 2003.

[4] David Beaver and Cleo Condoravdi. A uniform analysis of before and after. In Rob Young and Yuping Zhou, editors, Proceedings of SALT 13, Cornell university, 2003, to appear.

[5] Max Cresswell. Adverbial Modification. Reidel, Dordrecht, 1985.

[6] Haskell B. Curry and Robert Feys. Combinatory Logic: Vol. 1. North Holland, Amsterdam, 1958.

[7] Regine Eckardt. Event semantics. In Fritz Hamm and Ede Zimmermann, editors, Linguistiche Berichte Sonderheft (Semantics), pages 91-127. Helmut Buske Verlag, 2002.

[8] M. Enc. Towards a referential analysis of temporal expressions. Linguistics and Philosophy, 9, 1986.

[9] Nissim Francez and Michel Kaminski. An unbinding operator and its use in semantics of preposition-phrase modification. In Proceedings of Events04: Event Structures in Linguistic Form and Interpretation, Leipzig, Germany, March 17-19 2004. To appear.

[10] L.T.E. Gamut. Intensional logic and logical grammar(Vol. 2). The university of Chicago press, 1991.

[11] Michael Geis. Adverbial coordinate clauses in English. PhD thesis, MIT, 1970.

[12] Anastasia Giannakidou. Liscencing and sensitivity in polarity items: from downward entailment to (non)veridicality. Chicago Linguistic Society, 38, 2002.

[13] Anastasia Giannakidou and Frans Zwarts. Temporal, aspectual operators and (non)veridicality. In A. Giorgi, J. Higginbotham, and F. Pianesi, editors, The Syntax and Semantics of Tense and Mode Selection. Oxford University Press. To appear.

[] Jeroen Groenendijk and Martin Stokhof. Dynamic predicate logic. Linguistics and Philosophy, 14(1):39-101, 1996.

[14] Mark Hepple. The Grammar of Processing Order and Dependency: A Categorial approach. PhD thesis, University of Edinburgh, 1990.

[15] Norbert Hornstein. Towards a theory of tense. Linguistic Inquiry, 8:521-557, 1977.

[16] Aravind Joshi, K. Vijay-Shankar, and David Weir. The convergence of mildly context-sensitive formalisms. In Peter Sells, Stuart Shieber, and Tom Wasow, editors, Processing of Linguistic Structure. MIT Press, Cambridge, MA, 1991.

[17] Joachim Lambek. The mathematics of sentence structure. Amer. Math. monthly, 65:154-170, 1958.

[18] David Lewis. General semantics. Synthese, 22:18-67, 1970.

[19] Michael Moortgat. Categorial type logics. In Johan van Benthem and Alice ter Meulen, editors, Handbook of Logic and Language, pages 93-178. North Holland, 1997.

[20] Glyn V. Morrill. Type Logical Grammar: Categorial Logic of Signs. Kluwer Academic Publishers, 1994.

[] Reihard Muskens. Meaning and Partiality. CSLI publications, Stanford, CA, 1995. 
[21] Jong Park. Quantifier scope and constituency. In Proceedings of the 33rd annual meeting of the Association for Computational Linguistics (ACL), pages 205-212, San-Francisco, CA, 1995. Morgan Kaufman.

[22] Barbara Partee and Mats Rooth. Generalized conjunction and type ambiguity. In Rainer Baüerle, Christoph Schwartze, and Arnim von Stechow, editors, Meaning, use and interpretation of language. De Gruyter, Berlin, 1983.

[23] Barbara H. Partee. Some structural analogies between tense and pronouns in english. Journal of Philosophy, 70:601-609, 1973.

[24] Ian Pratt and Nissim Francez. On the semantics of temporal preposition phrases. Technical report, Dept. of computer science, university of Manchester, 1997. UMCS-97-4-2.

[25] Ian Pratt and Nissim Francez. Temporal prepositions and temporal generalized quantifiers. Linguistics and Philosophy, 24:187-222, 2001.

[26] Arnim Von Stechow. Temporal preposition phrases with quantifiers: Some additions to pratt and francez (2001). Linguistics and Philosophy, 25:755-800, August 2002.

[27] Mark Steedman. Surface Structure and Interpretation. MIT Press, Linguistic Inquiry monographs 30, 1996.

[28] Mark Steedman. Temporality. In Johan van Benthem and Alice Ter Meulen, editors, Handbook of Logic and Language, pages 895-938. North Holland, Amsterdam, 1997.

[29] Mark Steedman. The syntactic process. MIT Press, 2000.

[30] Mark Steedman. Scope alternation and the syntax-semantics interface. Ms., Univ. of edinburgh, 2002. URL http://www.cogsci.ed.ac.uk/ steedman/papers.html.

[31] Joost zwarts and Yoad Winter. Vector space semantics: a modeltheoretic analysis of locative prepositions. Journal of Logic, Language and Computation, pages 171 - 213, 2000. 